\title{
Brain-Derived Neurotrophic Factor Promotes Long-Term Potentiation-Related Cytoskeletal Changes in Adult Hippocampus
}

\author{
Christopher S. Rex, ${ }^{1}$ Ching-Yi Lin, ${ }^{2}$ Eniko A. Kramár, ${ }^{3}$ Lulu Y. Chen, ${ }^{2}$ Christine M. Gall, ${ }^{1,2}$ and Gary Lynch ${ }^{3}$ \\ Departments of ${ }^{1}$ Neurobiology and Behavior, ${ }^{2}$ Anatomy and Neurobiology, and ${ }^{3}$ Psychiatry and Human Behavior, University of California, Irvine, Irvine, \\ California 92697-4292
}

\begin{abstract}
Brain-derived neurotrophic factor (BDNF) is an extremely potent, positive modulator of theta burst induced long-term potentiation (LTP) in the adult hippocampus. The present studies tested whether the neurotrophin exerts its effects by facilitating cytoskeletal changes in dendritic spines. BDNF caused no changes in phalloidin labeling of filamentous actin (F-actin) when applied alone to rat hippocampal slices but markedly enhanced the number of densely labeled spines produced by a threshold level of theta burst stimulation. Conversely, the BDNF scavenger TrkB-Fc completely blocked increases in spine F-actin produced by suprathreshold levels of theta stimulation. TrkB-Fc also blocked LTP consolidation when applied 1-2 min, but not $10 \mathrm{~min}$, after theta trains. Additional experiments confirmed that p21 activated kinase and cofilin, two actin-regulatory proteins implicated in spine morphogenesis, are concentrated in spines in mature hippocampus and further showed that both undergo rapid, dose-dependent phosphorylation after infusion of BDNF. These results demonstrate that the influence of BDNF on the actin cytoskeleton is retained into adulthood in which it serves to positively modulate the time-dependent LTP consolidation process.
\end{abstract}

Key words: consolidation; synaptic plasticity; actin; phalloidin; cofilin; PAK

\section{Introduction}

The neurotrophins are well known to enhance neuronal survival and stimulate process outgrowth during development (Barde, 1994; Huang and Reichardt, 2001). These effects involve both global actions on the cell, via regulation of gene expression, and local modifications to axonal and dendritic cytoskeletons (Miller and Kaplan, 2001, 2003; Gehler et al., 2004). The neurotrophins retain strong regulatory control over neuronal survival, gene expression, and function in the mature nervous system ( $\mathrm{Lu}$ et al., 2005), and there are reasons to suspect that they also continue to exert discrete, local effects on the cytoskeleton. Specifically, naturalistic theta pattern afferent stimulation causes both the release of brain-derived neurotrophic factor (BDNF) (Balkowiec and Katz, 2002; Aicardi et al., 2004; Nagerl et al., 2004) and rapid modifications to spine actin networks in adult hippocampal slices (Lin et al., 2005a; Kramar et al., 2006). Possibly, these events are connected, with BDNF being released into mature synapses during theta activity and exerting local effects on the actin cytoskeleton comparable with those seen during development. Local actions could explain the strong positive influence of BDNF on

Received Sept. 15, 2006; revised Feb. 10, 2007; accepted Feb. 10, 2007.

This work was supported by National Institutes of Neurological Disorders and Stroke Grants NS051823, NS45260, and NS37799. C.S.R. was supported by National Institutes of Aging Grant AG00358. Use of the Bio-Rad Radiance 2000 confocal microscope system was provided by the University of California, Irvine Institute for Brain Aging and Dementia.

Correspondence should be addressed to Christine M. Gall, Gillespie Neuroscience Research Facility, University of California, Irvine, Irvine, CA 92697-4292. E-mail: cmgall@uci.edu.

DOI:10.1523/JNEUROSCI.4037-06.2007

Copyright $\odot 2007$ Society for Neuroscience $\quad$ 0270-6474/07/273017-13\$15.00/0 long-term potentiation (LTP) (Kang et al., 1997; Chen et al., 1999) because there is increasing evidence that activitydependent changes to the spine cytoskeleton stabilize the potentiation effect (Kramar et al., 2006).

We tested the above hypothesis in the present experiments. Previous studies applying rhodamine-labeled phalloidin, a mushroom toxin that binds filamentous actin (F-actin), to fixed tissue showed that intense afferent stimulation or direct glutamate receptor activation modifies the actin network in dendritic spines of dissociated cells (Fischer et al., 1998; Matus, 2000; Ackermann and Matus, 2003) and organotypic cultures (Okamoto et al., 2004) and increases regional F-actin levels in association with hippocampal LTP in vivo (Fukazawa et al., 2003). A limitation of this approach for studies of adult brain is imposed by generally high levels of phalloidin labeling, which make it difficult to identify changes in discrete structures such as secondary dendritic branches or spines amid the well labeled neuropil. To obviate this, we introduced a technical variant in which phalloidin is applied to living tissue in advance of fixation (Lin et al., 2005a). This in situ labeling procedure has made it possible to demonstrate that LTP induction in adult hippocampus causes marked F-actin increases within individual spines located in dendritic domains containing potentiated synapses (Lin et al., 2005a). The threshold levels of theta burst stimulation (TBS) needed to produce this effect correspond with those for induction of LTP, and manipulations that reverse potentiation also eliminate increases in F-actin (Kramar et al., 2006). Moreover, the newly assembled actin structures become resistant to disruption 
over the same time course as does potentiation (Kramar et al., 2006). Thus, in situ phalloidin labeling provides a means for testing the degree to which select variables affect cytoskeletal changes associated with LTP.

The hypothesis that BDNF facilitates potentiation by enhancing TBS-induced changes to spine actin networks predicts that exogenous BDNF will promote, and BDNF antagonists will block, increases in F-actin elicited by theta stimulation. It also requires BDNF signaling to the actin cytoskeleton to be present and operational in adult spines. The present studies confirm each of these predictions.

\section{Materials and Methods}

All animal procedures were conducted in accordance with the National Institutes of Health Guide for the Care and Use of Laboratory Animals and with protocols approved by the Institutional Animal Care and Use Committee of the University of California at Irvine.

Slice preparation and electrophysiological recordings. Transverse hippocampal slices $(350 \mu \mathrm{m})$ were prepared from young adult $(30-40 \mathrm{~d}$ old) male Sprague Dawley rats (Charles River Laboratories, Wilmington, MA) as described previously and maintained in an interface recording chamber perfused with artificial CSF (aCSF) $\left(60-70 \mathrm{ml} / \mathrm{h}, 31 \pm 1{ }^{\circ} \mathrm{C}\right)$ (Rex et al., 2005, 2006). Slices were allowed to equilibrate in the recording chamber for 2-3 h before recordings or other treatments were initiated. Unless otherwise noted, chemicals were purchased from Sigma (St. Louis, MO).

Recordings of field EPSPs (fEPSPs) were collected from CA1b stratum (str.) radiatum using a single glass pipette $(5 \mathrm{M} \Omega, 2 \mathrm{M} \mathrm{NaCl})$. Bipolar stimulation (twisted nichrome wires) was delivered at two sites (CAla and CA1c) in the apical Schaffer collateral projections (supplemental Fig. $1 A, B$, available at www.jneurosci.org as supplemental material). For tests of CA1 basal dendritic evoked responses, a similar stimulation/ recording protocol was used in str. oriens. Single-pulse baseline stimulation was delivered at $0.05 \mathrm{~Hz}$ for $20-30 \mathrm{~min}$ as described previously (Arai et al., 1994; Rex et al., 2005, 2006). Slopes and amplitudes of fEPSPs were measured from digitized files (NacGather 2.0; Theta Burst, Irvine, CA) and normalized to the last $10 \mathrm{~min}$ of the baseline period; all measures are reported and illustrated as group means \pm SEM. Synaptic potentiation was induced using TBS (bursts of four pulses at $100 \mathrm{~Hz}$ separated by 200 ms; unless otherwise noted, trains of 10 bursts were applied) (Larson et al., 1986; Rex et al., 2005). TBS applied to phalloidin-treated slices was delivered to the two stimulation electrodes within $1 \mathrm{~min}$ of each other, and slices were collected $8-15 \mathrm{~min}$ after the second stimulation; control slices continued to receive baseline stimulation for an equivalent period. Responses to individual theta bursts within the train were recorded and analyzed as the burst response area below baseline level. To evaluate response facilitation within the train, the sizes (composite area) of responses to bursts 2-10 were expressed as percentage change from the composite area of the initial burst response (Kramar et al., 2004).

Extracellular application of phalloidin and drugs. Fluorescent-tagged phalloidin $(2-4 \mu \mathrm{l})$ was applied topically from a micropipette every 5 min over a period of $20 \mathrm{~min}$ (supplemental Fig. $1 \mathrm{D}$, available at www. jneurosci.org as supplemental material) either (1) after a 2-3 h stabilization period before TBS or (2) at a range of time points after TBS. Rhodamine-phalloidin was purchased from Sigma or Invitrogen (Carlsbad, CA). The Sigma product was handled as described previously (Lin et al., 2005a); the Invitrogen product was diluted in $\mathrm{ddH}_{2} \mathrm{O}$ to $12 \mu \mathrm{M}$. Stocks were stored at $-20^{\circ} \mathrm{C}$ and diluted in aCSF to the working pipette concentration of $6 \mu \mathrm{m}$ each day. Although it is reported that phalloidin does not penetrate cells (Cooper, 1987), different groups have provided evidence that it is membrane permeant (Bernstein et al., 1998; Geeraert et al., 2003; Lin et al., 2005a; Kramar et al., 2006), and discrete spine labeling was detected with extracellular application in the present studies.

Some experiments used pipette coapplication of drugs and rhodamine-phalloidin. In these cases, drug stocks $\left(-20^{\circ} \mathrm{C}\right)$ were diluted to final working concentrations along with phalloidin in aCSF. Latrunculin A (Invitrogen) stock was prepared in DMSO $(<0.05 \%$ in final solution), whereas BDNF (Chemicon, Temecula, CA), DL-2-amino-5- phosphonovaleric acid (APV) (Sigma), and K252a (Calbiochem, San Diego, CA) were prepared in $\mathrm{ddH}_{2} \mathrm{O}$. The major experiments using BDNF were repeated with the same concentration of BDNF that had been boiled (10 $\mathrm{min}$ ) before experimentation to inactivate the protein (Scharfman, 1997). In distinction from the active neurotrophin, boiled BDNF had no effect on phalloidin labeling of spine profiles with TBS.

Bath infusion of latrunculin A was performed via a second infusion line attached to the main input line. Preliminary studies determined that 500 nм latrunculin A did not significantly alter baseline synaptic potentials or input-output curves after 30 min bath application (our unpublished observations).

The recombinant TrkB-Fc was used to sequester extracellular BDNF (and potentially the far less abundant neurotrophin 4) and thereby interfere with BDNF signaling in the slice (Shelton et al., 1995). Stock TrkB-Fc or control IgG-Fc (R \& D Systems, Minneapolis, MN) was prepared in Tris-buffered saline containing $0.1 \%$ bovine serum albumin and diluted to working concentrations in aCSF. For rapid infusion of these compounds, a drug-filled glass micropipette (tip diameter of $\sim 25$ $\mu \mathrm{m}$ ) attached to a $10 \mathrm{ml}$ syringe was lowered by micromanipulator into the bath upstream from the recording site. Flow from the pipette $(0.2$ $\mathrm{ml} / \mathrm{min}$ ) was controlled by a motorized injection pump (Sage Instruments, Freedom, CA). The concentration of TrkB-Fc or IgG-Fc in the micropipette was $10 \mu \mathrm{g} / \mathrm{ml}$ (estimated five times higher than the final desired concentration) to compensate for dilution in the slice chamber.

Immunocytochemistry. Because of relatively weak binding between phalloidin and F-actin, we found that phalloidin labeling was markedly reduced with even relatively short incubations $[2-4 \mathrm{~h}$, room temperature (RT)] in detergent-containing buffers used for immunocytochemistry. Therefore, to compensate, slices intended for immunostaining were labeled with $12 \mu \mathrm{M}$ AlexaFluor 568-conjugated phalloidin (Invitrogen) in the manner described above. After phalloidin application and electrophysiological recording, slices were fixed in $4 \%$ paraformaldehyde in 0.1 M sodium phosphate buffer (PB) for $12-16 \mathrm{~h}$, cryoprotected in $20 \%$ sucrose $/ \mathrm{PB}$ for $2 \mathrm{~h}$ at $4^{\circ} \mathrm{C}$, sectioned on a freezing microtome at $20 \mu \mathrm{m}$, and then mounted onto Fisher Superfrost Plus slides (Fisher Scientific, Pittsburgh, PA). Sections were then incubated ( $1 \mathrm{~h}, \mathrm{RT})$ in mouse antipostsynaptic density 95 (PSD-95) (MAB1598; Chemicon) at 1:100 in 0.1 M PB containing $4 \%$ bovine serum albumin and $0.3 \%$ Triton X-100 (PBT). Slides were then washed in PB (three times for $5 \mathrm{~min}$ ), incubated (45 min, RT) with fluorescein anti-rabbit IgG (1:200; Vector Laboratories, Burlingame, $\mathrm{CA}$ ) in $\mathrm{PBT}$, rinsed in $\mathrm{PB}$, and coverslipped with Vectashield mountant (Vector Laboratories). Although we obtained specific PSD-95 labeling with these procedures, comparisons with longer antibody incubations indicate that this protocol underestimates numbers of PSD-95-immunoreactive puncta (C. S. Rex and C. M. Gall, unpublished observations).

For p21 activated kinase 3 (PAK3) and PSD-95 double immunostaining, hippocampal slices $(350 \mu \mathrm{m})$ were prepared as described above for electrophysiology and maintained in oxygenated aCSF for 1-2 h. Fixation and sectioning are described above. Slide-mounted sections were incubated for $40 \mathrm{~h}\left(4^{\circ} \mathrm{C}\right)$ in mouse anti-PSD-95 (1:1000; Chemicon) and rabbit anti-PAK3 (1:500; catalog \#06-902; Upstate, Charlottesville, VA) in PBT. Slides were then washed in PB and incubated $(1 \mathrm{~h}$, room temperature) in a mixture of Texas Red anti-rabbit IgG and fluorescein anti-mouse IgG (1:200 in PBT; Vector Laboratories) to label PAK3 and PSD-95 antisera, respectively. After final rinses, slides were coverslipped with Vectashield. Control tissue was processed by the same procedures but with exclusion of one or the other primary antisera from the initial incubation step: under these conditions, the only labeling observed was associated with the primary antibody included in the initial incubation, thereby demonstrating the species specificity of secondary antisera.

Imaging and measures of dendritic spines. From preliminary studies (supplemental Fig. 2, available at www.jneurosci.org as supplemental material), we determined that the superior detection efficiency achieved by a charge-coupled device (CCD) camera compared with laser-scanning confocal microscopy made wide-field microscopy coupled with parametric estimation signal processing the favorable choice for quantifying a broad range of label intensities (Swedlow et al., 2002). Rhodamine-phalloidin labeling was examined using epifluorescence illumination and a 
Zeiss (Oberkochen, Germany) Axioscope microscope equipped with an Axiocam CCD camera and AxioVision 3.1 software. For quantitative analysis, digital images were collected at $20 \times$ magnification from three serial sections situated $20-80 \mu \mathrm{m}$ below the surface of the hippocampal slice. For each field, a series of 16-20 digital photomicrographs were collected at regular focal steps on the $z$-axis. Camera exposure times were adjusted for each experiment so that $\sim 6-12$ spines could be visualized in the image field in control slices. All images intended for comparison were then collected using the same exposure intervals.

Digital $Z$-plane image stacks were collapsed by extended focal imaging (see Fig. $2 \mathrm{~A}$ ) into a single image using Microsuite FIVE (Soft Imaging Systems, Lakewood, CO). These images were then converted to grayscale, and intensity levels were scaled to values determined for each experiment (Adobe Photoshop 6.0; Adobe Systems, Mountain View, CA) to visualize low-intensity labeling. Labeled spines were measured and counted from a $550 \mu \mathrm{m}^{2}$ sampling zone in the proximal str. radiatum between the stimulating electrodes (supplemental Fig. $1 B$, available at www.jneurosci.org as supplemental material). Analysis was conducted blind to sample identity on batches of slices that had been sectioned and stained together. In-house-built software (Lin et al., 2005a; Kramar et al., 2006) was used to count labeled spine-like structures. Briefly, intensity thresholds ( 8 bits/pixel) were applied to identify spine-like structures at varying levels of label intensity within a range that reliably counted dendritic spines (see Fig. $2 \mathrm{~B}$ ). Pixel values for each image were normalized to reduce the impact of background intensity differences across the image, binarized using each intensity threshold, and finally cleaned by "erosion" and "dilation" filtering (Jain, 1984). Object area and eccentricity were determined from this method and used to identify observations as spines or artifact. Digital images of objects identified as spines were overlaid semi-transparently with the original photomicrographs to verify accuracy. Spine counts from each of the three serial sections were averaged to produce a representative value for each slice. Unless otherwise noted, population counts represent numbers of slices.

Spine label intensity distributions were created for each group. Distributions for control and TBS-treated slices were significantly different from "normal" $[p<0.001$, two-tailed one-sample Kolmogorov-Smirnov (K-S) test for both groups]. Therefore, group comparisons used the nonparametric two-sample $\mathrm{K}-\mathrm{S}$ test. In figures, cumulative plots show the cumulative proportion of the total number of identified spines with labeling intensity values that exceeded a given threshold (from high to progressively lower labeling intensity values). To account for independent samples, significance across the range of label intensity values was assessed by two-way repeated-measures ANOVA of spine counts within 10 pixel intensity unit (PIU) bins. From initial studies, we determined that identified spine structures with label intensities $>145$ PIUs reliably showed TBS-dependent effects; therefore, counts of "densely labeled" spines described throughout the present report used thresholding at the 145 PIU level for TBS versus non-TBS group comparisons. Independent samples collected under these conditions exhibited normal distributions; therefore, significance of effects of treatment on high-intensity spine labeling was determined by either the two-tailed $t$ test or the one-way ANOVA and post hoc Tukey's honestly significant difference (HSD) test.

Laser scanning confocal microscopy. Laser scanning confocal microscopy was performed using the Bio-Rad (Hercules, CA) Radiance 2000 Laser Scanning System; phalloidin/antigen double-fluorescent labeling was assessed with Bio-Rad Lasersharp 2000 software. Optical sections $(1.0 \mu \mathrm{m})$ acquired with a PlanApo $60 \times$ oil objective were scanned in a $512 \times 512$ pixel format. Tissue sections were qualitatively analyzed from image $Z$-stacks, and image processing applied uniformly to the entire batch was performed with Photoshop CS (version 8.0; Adobe Systems).

Double-fluorescent immunolabeling for PAK3 and PSD-95 was captured using a Zeiss LSM 510 META laser scanning system. Adjacent fields of hippocampal CA1 were acquired with a $60 \times$ PlanApo oil objective in a $1024 \times 1024$ pixel format and assembled in Photoshop. Double-label filtering applied to images to identify regions of overlap was performed by linear match of 8-bit level scale to yellow saturation (Matlab; MathWorks, Novi, MI). Quantitative analyses of PAK3, PSD-95, and doublelabeled puncta were performed as described above for spine analysis. For all confocal imaging, tests for emission and excitation crosstalk were performed at the beginning of each acquisition session.

Western blot analysis of BDNF-treated slices. Acute hippocampal slices (400 $\mu \mathrm{m}$ thick) were prepared from young adult male (3-5 week) Sprague Dawley rats using a Mcllwain tissue chopper. Slices were maintained $\left(32 \pm 1^{\circ} \mathrm{C}\right)$ on polymer membrane inserts in a static aCSF bath with upper surfaces exposed to $95 \% \mathrm{O}_{2}-5 \% \mathrm{CO}_{2}$. Slices were given $1 \mathrm{~h}$ recovery after preparation before the aCSF was replaced with the same solution including $1 \mathrm{mM} \mathrm{Na}_{3} \mathrm{VO}_{4}$ (phosphatase inhibitor) and specified concentrations of BDNF, TrkB-Fc, and/or IgG-Fc. Bath aCSF was washed over the tissue every 15-20 min during recovery, with treatment, and every 15-20 min thereafter. After treatment, slices were homogenized in radioimmunoprecipitation assay buffer (Lin et al., 2005b) containing Phosphatase Inhibitor Cocktails 1 and 2 (P2850 and 5726) and Complete Protease Inhibitor Cocktail (Roche Diagnostics, Indianapolis, IN).

Sample protein levels were measured (Bio-Rad Protein Assay), and volumes were adjusted to normalize microgram per microliter protein content. Proteins were then separated by $4-20 \%$ SDS-PAGE $(100 \mu \mathrm{g} /$ lane for cofilin and $40 \mu \mathrm{g} / \mathrm{lane}$ for PAK) and processed for Western blot analysis of immunoreactivity (IR) for cofilin (1:1500; ab11062-50; Abcam, Cambridge, MA), phospho-cofilin (pS3) (1:1500; ab12866-50; Abcam), PAK3 (1:1500; 06-902; Upstate), and phospho-PAK1/2/3 (pS141) (1:1500; 44-940G; Invitrogen) using the ECL Plus chemiluminescence system (Amersham Biosciences, Arlington Heights, IL). Levels of immunoreactivity were assessed by densitometric analysis of films using the AIS system (Imaging Research, St. Catharine's, Ontario, Canada) as described previously (Lin et al., 2005b). Statistical significance was determined by one-way ANOVA and Tukey's HSD post hoc test.

\section{Results}

\section{Theta bursts cause actin polymerization in adult dendritic spines}

Hippocampal slices from 30 - to 45 -d-old rats were incubated with rhodamine-phalloidin for $20 \mathrm{~min}$, tested for physiology over the next 15-20 min, and then fixed. Supplemental Figure $1 C$ (available at www.jneurosci.org as supplemental material) shows that evoked responses (set to $40-50 \%$ maximum amplitude) recorded within the period after topical phalloidin application had normal amplitudes and shapes for fEPSPs in str. radiatum. LTP was induced by staggered delivery of TBS to two convergent populations of Schaffer-commissural fibers. The $6 \mu \mathrm{M}$ concentrations of topically applied phalloidin used here are $\sim 5 \%$ of those reported to attenuate LTP (Kim and Lisman, 1999). Potentiation in the presence of the marker was robust and of the same magnitude ( $149 \pm 10 \%$ at 15 min after TBS; mean \pm SEM $)$ as described in the literature for otherwise untreated slices. There were no evident phalloidin-related changes in theta burst responses or in their facilitation during the theta train (supplemental Fig. $1 E, F$, available at www.jneurosci.org as supplemental material).

Tests for LTP-related increases in F-actin labeling used microscopic luminescence/detection levels set to identify $6-12$ brightly labeled spine-like profiles within a $550 \mu \mathrm{m}^{2}$ str. radiatum sampling area in control slices. Figure $1 A$ shows labeling in field CA1 of slices that received theta burst compared with low-frequency stimulation of converging Schaffer-commissural afferents. TBS resulted in the appearance of greater numbers of intensely labeled, punctate structures of $\sim 0.5-1 \mu \mathrm{m}$ in diameter, many of which were aligned with, but slightly displaced from, the surface of dendrites (Fig. $1 \mathrm{~A}, \mathrm{Bi}$, Biii). Other puncta occurred in isolation, adjacent to dendrites that were only very lightly labeled (Fig. 1 Bii). More faintly labeled structures of similar size and shape could be readily detected in control slices (Fig. 1 A, Biv). The size, location, and appearance of the phalloidin-labeled profiles indicated that they were spine heads.

F-actin localization was also examined by combining in situ 
phalloidin labeling with postfixation immunostaining for PSD-95, a marker for excitatory synapses (Hunt et al., 1996). Preliminary studies showed that immunocytochemical procedures attenuated rhodamine-phalloidin labeling, but this problem was overcome with a more robust fluorochrome (Alexa 568-phalloidin) and shorter incubation times. Double labeling was assessed in thin optical sections $(1 \mu \mathrm{m})$ using laser scanning confocal microscopy. Under these conditions, PSD-95-IR appeared as 0.5- to $1-\mu \mathrm{m}$ diameter discs scattered across the dendritic layers of field CA1 (Fig. 1D). Many phalloidin-labeled profiles in the region surrounding the physiological recording site were associated with PSD-95-IR (Fig. $1 C-E)$ : In some instances, the PSD-95immunopositive discs could be seen to "cap" the head of phalloidin-labeled spines, whereas in others they were superimposed on the F-actin labeling (Fig. $1 E-H)$.

Photomicrographs of the region between the two stimulating electrodes revealed labeled spines across a range of fluorescence intensities. After $Z$-series image capture and signal processing (Fig. 2A), image gray values were scaled to closely match the range of labeling intensities within a given experiment, and then spine-like profiles were identified and quantified using an image scanning system (Fig. $2 B$ ). Figure $2 C$ describes typical quantification of intensely labeled spines ( $\geq 145$ PIU labeling threshold; see Materials and Methods) when rhodamine-phalloidin was applied for $20 \mathrm{~min}$ before delivery of TBS. Additional experiments evaluated labeling with phalloidin applied to slices for $20 \mathrm{~min}$ beginning $30 \mathrm{~s}(n=3)$, $20 \mathrm{~min}(n=3)$, and $60 \mathrm{~min}(n=2)$ after the delivery of TBS (Fig. $2 C$ ). The numbers of labeled profiles obtained with these different phalloidin treatment schedules were not different from those obtained with pre-TBS application of the marker: in each case, the incidence of phalloidin-

labeled puncta was significantly greater in TBS compared with paired control (not stimulated) slices. This result indicates that the observed effects do not reflect TBS-induced entry of phalloidin into spines.

The effects of TBS were primarily restricted to the proximal and middle str. radiatum (Fig. $3 A, B$ ), the dendritic lamina containing the potentiated synapses (Shimono et al., 2002) (Fig. 3C). Analyses of the frequency of intensely labeled spines ( $>145$ PIU) within field CA1 indicated that their incidence was twofold to fourfold greater in the stimulated zone (midproximal str. radiatum) than in neighboring regions (Fig. $3 D, E)$. APV $(100 \mu \mathrm{M})$, an antagonist of the voltage-sensitive NMDA receptor, did not by itself influence the spine label intensity distribution (Fig. $3 \mathrm{H}$, top graph) (see below) but did block TBS-induced increases in phal-
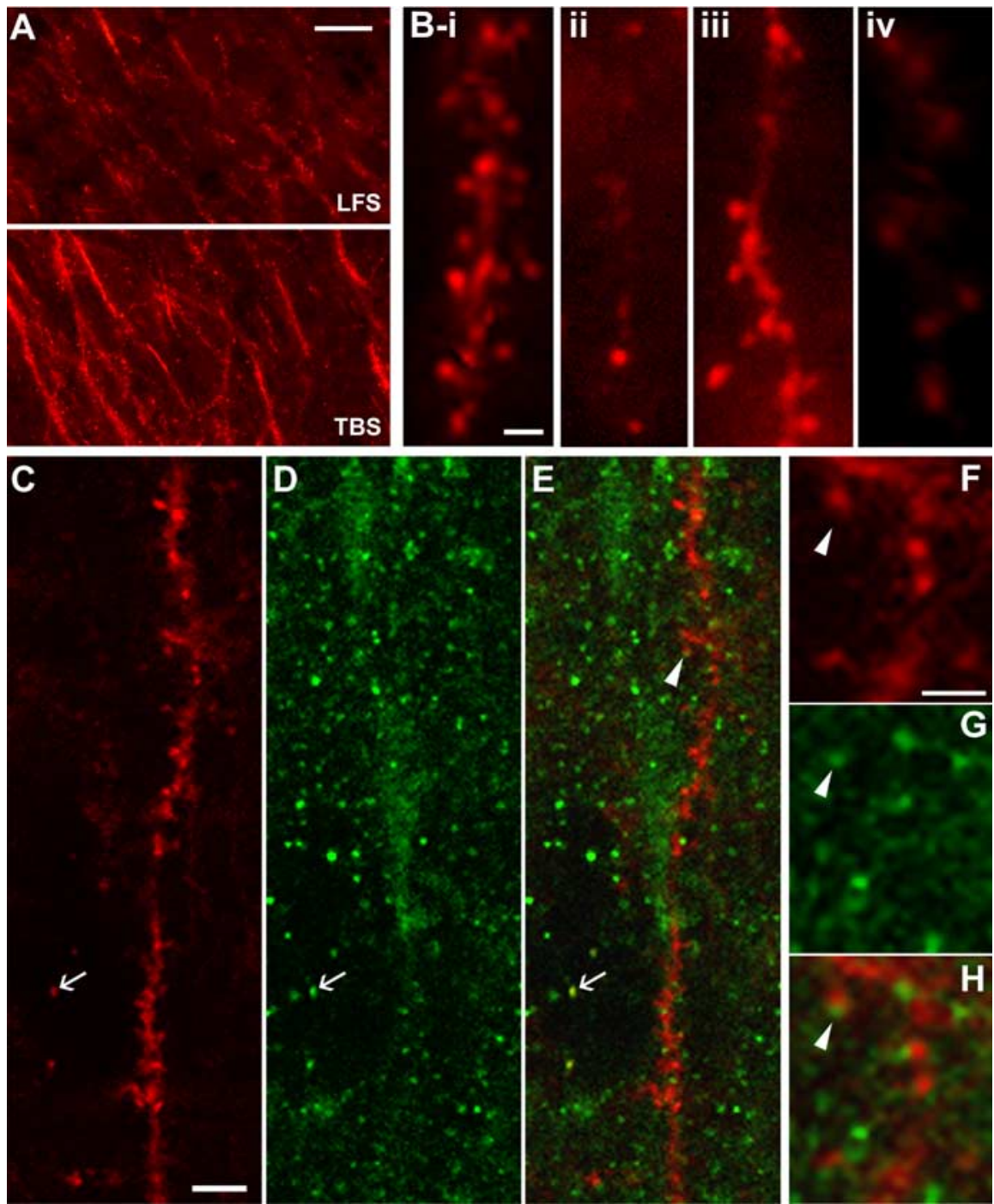

Figure 1. Theta burst stimulation increases F-actin labeling in adult dendritic spines. $\boldsymbol{A}$, Low-power photomicrographs of -actin labeling in field CA1 of hippocampal slices that received topical rhodamine-phalloidin followed by either low-frequency 列 (n) $\boldsymbol{F}$-actin-positive spine (arrowhead in $\boldsymbol{E}$ ) or superimposition of the two labels (see arrow in $(-\boldsymbol{E})$. $\boldsymbol{F}-\boldsymbol{H}$ show the region around the arrowhead in $\boldsymbol{E}$ at higher magnification (arrowhead indicates same location in $\boldsymbol{E}-\boldsymbol{H}$ ) to illustrate the PSD-95 capping effect. Scale bars: (in $\boldsymbol{C}$ C, $\boldsymbol{D}, 5 \mu \mathrm{m}$; (in $\boldsymbol{F}) \boldsymbol{F}-\boldsymbol{H}, 2 \mu \mathrm{m}$.

loidin labeling (K-S test, $D=0.16 ; p<0.001$ ) (Fig. $3 G, H$, bottom graph), indicating that the densely labeled spines were located on functioning neurons.

We next compared the fluorescence intensity distribution of labeled puncta in control and TBS cases to estimate the percentage of F-actin-containing spines affected by stimulation. Photomicrographs of sections through control and experimental (TBS) slices, illuminated at the same intensity, are shown on the left of Figure 4, $A$ and $B$, with their corresponding pseudocolor images on the right (demonstrating variations in label intensity). Note that both sections contain numerous faintly labeled puncta, but only in the TBS case are there significant numbers of densely labeled profiles. Cumulative frequency plots of spines across the range of labeling intensity values (Fig. $4 D$ ) show that TBS signif- 

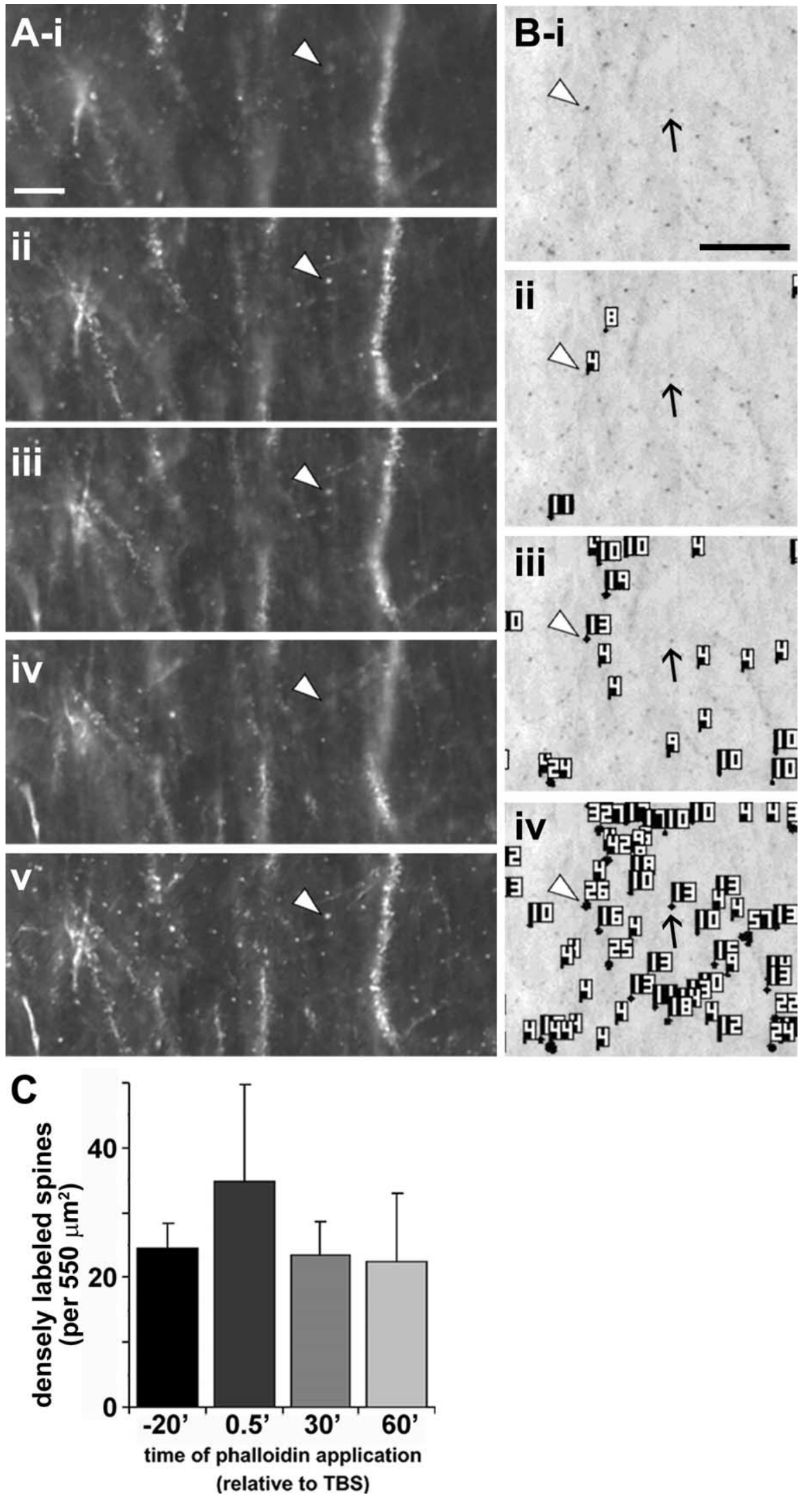

Figure 2. Spine detection analysis procedure for in situ rhodamine-phalloidin labeling. Panels illustrate the spine detection analytical technique for a representative phalloidin-labeled field. After experimental treatment (rhodamine-phalloidin infusion, stimulation), slices were fixed and sectioned at $20 \mu \mathrm{m}$ to assess dendritic spine labeling. $\boldsymbol{A}$, For each section analyzed, a series of wide-field photomicrographs were collected at regular, sequential $z$-axis planes $(\boldsymbol{i}-\boldsymbol{i v})$ and collapsed using extended focal imaging $(\boldsymbol{v})$ to eliminate out-of-focus noise and permit accurate spine quantification through the $z$-axis of the slice. Scale bar: (in $\boldsymbol{i}) \boldsymbol{i}-\boldsymbol{v}$ $10 \mu \mathrm{m} . \boldsymbol{B}$, Representative photomicrograph (i) shows collapsed Z-series images from a slice given only low-frequency stimulation. icantly changed the intensity distribution to favor high-intensity labeling ( $\mathrm{K}-\mathrm{S}$ test for TBS vs control, $D=0.25 ; p<0.0001)$ Significance between TBS-treated and control slices was also achieved when counts from individual slices were compared (two-way repeated-measures ANOVA, $p<0.01)$. Counts of all visibly labeled spine-like profiles (i.e., including all intensity values above 75 PI threshold) were comparable for control $(70 \pm 7)$ and stimulated ( $76 \pm 8 ; p>0.5$, two-tailed $t$ test) slices (Fig. $4 E$ ), but TBS-stimulated slices contained two to three times more of the most intensely labeled spines than did control slices $(29 \pm 5$ vs $12 \pm 3$, respectively, at 145 PIU threshold; $p<0.01$ ) (Fig. $4 F$ ). It thus appears that induction of LTP in two converging inputs converted $\sim 20 \%$ of the labeled spine population in the innervated dendritic zone from low to high F-actin content. This provides an upper limit for the percentage of spines affected by TBS.

That the increased labeling seen after TBS is attributable to actin polymerization was tested with latrunculin $\mathrm{A}$, a toxin that binds to G-actin and thereby prevents it from being assembled into filaments (Smart et al., 2003). Brief application of latrunculin A (500 nM) eliminated TBSinduced increases in spine labeling, as is evident in both photomicrographs taken with low levels of illumination (Fig. 4Ci) and pseudocolor representations (Fig. 4Cii). A plot of labeled spine number as a function of luminescent threshold (Fig. 4D) shows that latrunculin A applied with TBS shifted the intensity distribution away from densely labeled profiles compared with the distribution generated with TBS alone $(\mathrm{K}-\mathrm{S}$ test, $\mathrm{D}=0.33 ; \mathrm{p}<$ $0.0001)$ : the toxin completely blocked the induction of high-intensity spine labeling $(2 \pm 1$ at 145 PIU threshold; $p=0.001$,

\footnotetext{
$\leftarrow$

For spine analysis, image pixel intensity was inverted so that rhodamine-labeled elements appear as dark puncta. ii-iv show spine detection software output for three pixel intensity threshold levels (with decreasing thresholds from ii-iv): the analysis software identified greater numbers of spines as intensity threshold was lowered. Note that intensely labeled spines (e.g., spine at arrowhead in Bi) are detected, and identified with an object number, at all threshold levels, whereas faintly labeled spines (arrow) are only detected when the intensity filter is set very low (iv). Scale bar: (in $\boldsymbol{i}), \boldsymbol{i}-\boldsymbol{i} \boldsymbol{i}, 10 \mu \mathrm{m}$. C, Bar graph shows group mean \pm SEM counts of intensely labeled spine-like puncta within the $550 \mu \mathrm{m}^{2}$ sample region for hippocampal slices infused for 20 min with rhodaminephalloidin beginning $20 \mathrm{~min}$ before TBS $\left(-20^{\prime}\right)$ or $0.5,20$, or 60 min after TBS (spines detected at high threshold, 145 PIU levels only). There were no significant differences in labeled spine counts between groups ( $p>0.7$, one-way ANOVA).
} 
two-tailed $t$ test) (Fig. $4 F$ ) without significantly reducing the total number of labeled spines ( $67 \pm 10$ at 75 PIU threshold; $p>0.4$ ) (Fig. 4E). Latrunculin A applied alone reduced $\mathrm{F}$-actin labeling intensity from baseline conditions ( $\mathrm{K}-\mathrm{S}$ test for latrunculin A vs controls, $D=0.10 ; p<$ $0.01)$; this result, in conjunction with the lack of effect of the toxin on basal synaptic transmission, suggests that variations in F-actin levels in the absence of synaptic potentiation do not influence the postsynaptic response. Spine label intensity distributions were comparable for slices treated with latrunculin A alone or in combination with TBS (K-S test, $D=0.04 ; p>$ 0.05 ) indicating that TBS-induced activation of spine actin polymerization is ineffective in the presence of the drug. In agreement with previous results (Krucker et al., 2000; Lang et al., 2004), bath infusion of latrunculin A also disrupted the stabilization, but not the induction and initial expression, of LTP (Fig. 4G).

\section{BDNF promotes theta stimulation-} induced actin polymerization

If BDNF facilitates the formation of F-actin, then treatment with the exogenous neurotrophin should reduce the amount of TBS needed to produce effects of the kind described above. A test of this prediction is described in Figure 5. In accord with previous findings (Kramar et al., 2006), we found that stimulation with a single pair of theta bursts does not cause a statistically significant increase in the number of spines with high levels of phalloidin labeling in otherwise untreated slices (Fig. $5 A, B)(8.9 \pm 6.9$ vs $12.8 \pm 3.7$ for control vs two-burst TBS, respectively; $p>0.3$, two-tailed $t$ test). This stimulation protocol was effective, however, when delivered to slices that had been incubated with $2 \mathrm{~nm} \mathrm{BDNF}$ for $20 \mathrm{~min}$ (Fig. 5B) (35.1 \pm 1.7 densely labeled spines; $p<$ 0.01 vs TBS alone). This represents an approximate threefold increase in intensely labeled spines relative to what was obtained in control slices, confirming that BDNF substantially reduces the threshold for activity-driven increases in F-actin content. Importantly, treatment with BDNF in the absence of theta stimulation at concentrations ranging from 2 to $15 \mathrm{nM}$ did not increase numbers of spines with dense F-actin labeling (two-tailed $t$ tests, $p>0.2$ for 2, 10, and 15 nM BDNF vs controls).

In accord with previous works (Figurov et al., 1996; Frerking et al., 1998; Gottschalk et al., 1998; Kovalchuk et al., 2002; Kramar et al., 2004), we found that BDNF infusion does not measurably affect synaptic responses over $30 \mathrm{~min}$ of testing but nonetheless markedly reduces the stimulation requirements for producing
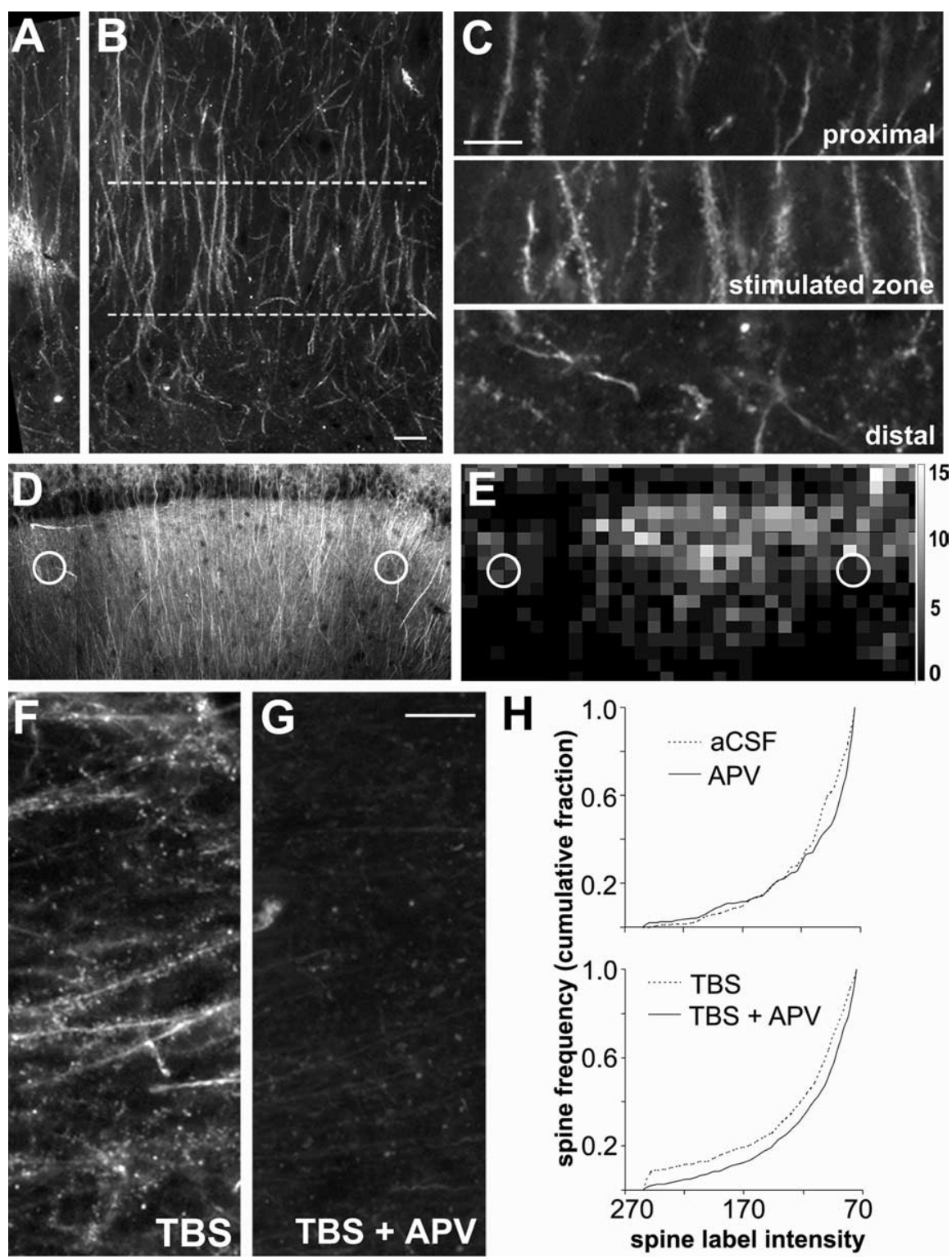

Figure 3. TBS-induced F-actin labeling is spatially restricted and blocked by an NMDA receptor antagonist. $\boldsymbol{A}, \boldsymbol{B}$, Low-power photomicrographs of CA1 str. radiatum in an adult hippocampal slice labeled with rhodamine-phalloidin in situ. Bright labeling in the middle of $A$ marks the location of the stimulation electrode ( $40-60 \mu \mathrm{m}$ below surface of slice) in the mid-distal portion of str. radiatum. The lamina aligned with the proximodistal position of the stimulation electrodes is outlined (between dashed lines) in $\boldsymbol{B}$. Scale bar: (in $\boldsymbol{B}) \boldsymbol{A}, \boldsymbol{B}, 10 \mu \mathrm{m}$. $\boldsymbol{C}$, Enlargements of the stimulated zone (middle) and adjacent proximal (top) and distal (bottom) regions from $\boldsymbol{B}$ show that robust spine labeling is restricted to lamina containing the stimulated afferents. Scale bar, $10 \mu \mathrm{m}$. $\boldsymbol{D}$, Photomicrograph of hippocampal field CA1 from a slice that received TBS; stimulation electrode placements are indicated by white circles. $\boldsymbol{E}$, Spatial frequency map plots the distribution of intensely labeled spine numbers (>145 PIU) across $10 \times 10 \mu \mathrm{m}$ sampling zones for the hippocampal field shown in $\boldsymbol{D}$. Intensely labeled spine frequencies are represented by grayscale intensity. Grayscale calibration bar (at right) shows number of spines per sampling region represented by the different gray levels. $\boldsymbol{F}, \mathbf{G}$, Photomicrographs of sample regions taken from phalloidin-labeled slices receiving TBS in the presence of aCSF alone (TBS) or together with $100 \mu \mathrm{m}$ APV (TBS + APV). Scale bar, $10 \mu \mathrm{m}$. $\boldsymbol{H}$, Top graph describes cumulative fraction of spine counts across the full range of label pixel intensities within the $550 \mu \mathrm{m}^{2}$ sampling zone for control unstimulated slices (aCSF, dashed line) versus slices treated with APV (solid line). Bottom graph plots same distribution for slices stimulated with TBS in aCSF (dashed line) compared with slices receiving TBS in the presence of APV (solid line).

LTP. A single pair of theta bursts produced only modest and short-lived potentiation in control slices but elicited a measurable degree of LTP in slices pretreated with $2 \mathrm{nM} \mathrm{BDNF}$ (Fig. 5C) $(117 \pm 7 \%$ of baseline at $15 \mathrm{~min}$ after induction vs $101 \pm 3 \%$ for untreated controls; $p<0.05$, two-way repeated-measures ANOVA for minutes 10-15).

We next asked whether endogenous BDNF released during 

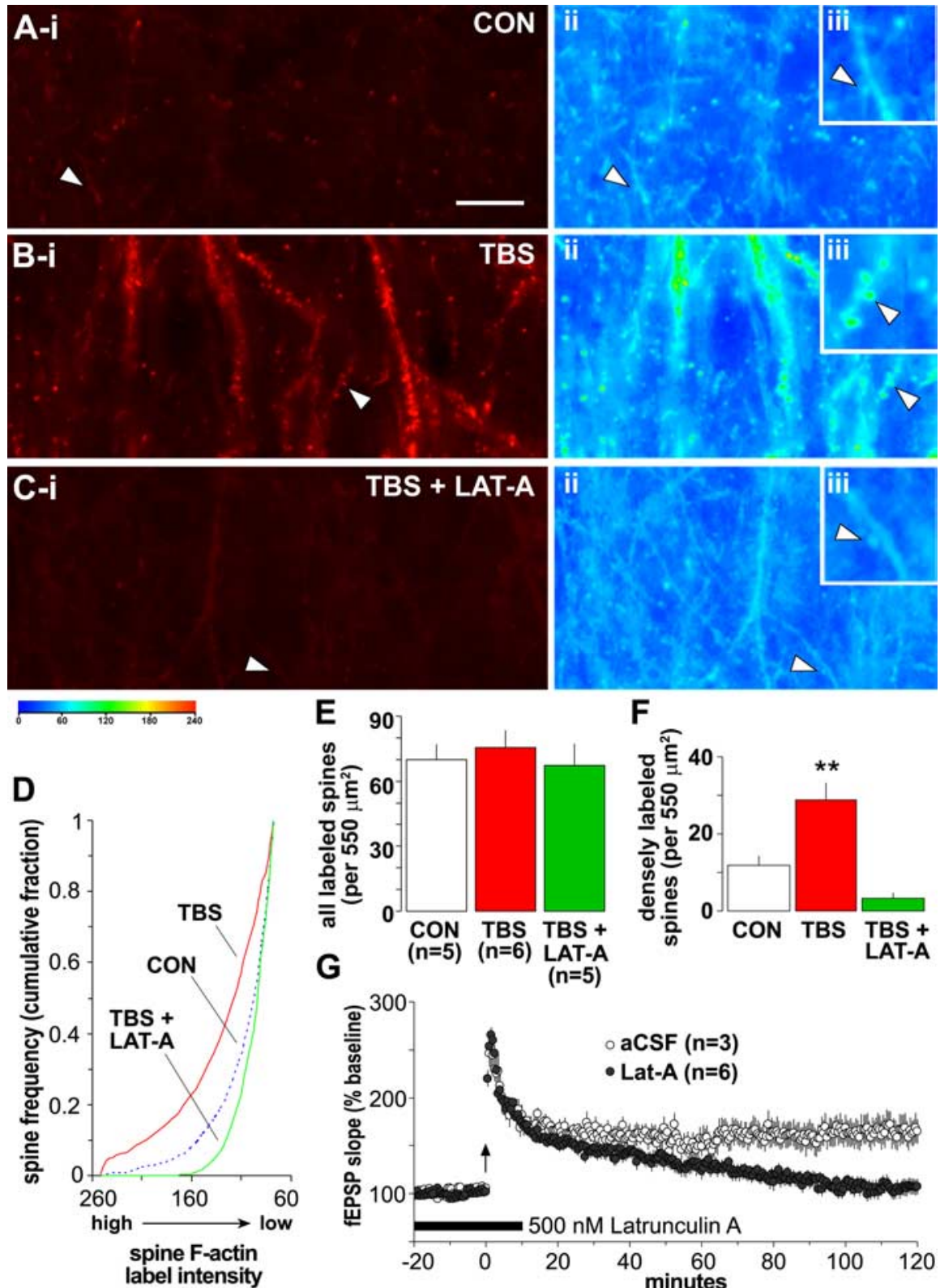

$\mathbf{F}$
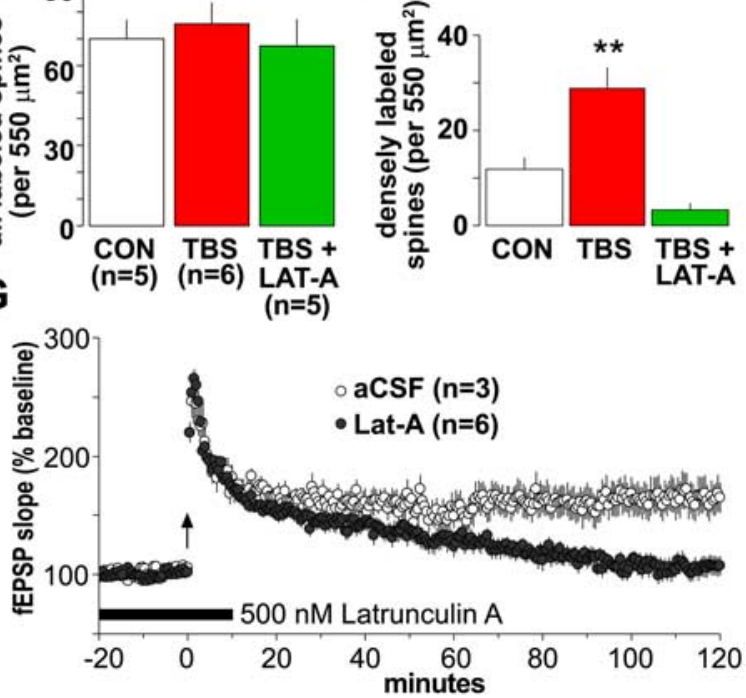

Figure 4. TBS-induced increases in the number of densely labeled spines are reversed by latrunculin A.A-C, Left, Photomicrographs of $550 \mu \mathrm{m}^{2}$ sample regions taken with low-intensity illumination, from in situ phalloidin-labeled slices that received either baseline stimulation (CON), TBS, or TBS in the presence of $500 \mathrm{~nm}$ latrunculin A (LAT-A). Note that TBS caused a marked increase in structures containing dense phalloidin labeling of F-actin and that this effect was eliminated by latrunculin A. Pseudocolor representations of label density (PIU calibration at bottom) for these three sections are shown to the right (ii and iii in $\boldsymbol{A}-\boldsymbol{C}$ ). The control (Aii) has numerous lightly labeled (blue end of spectrum) puncta that, at higher magnification (inset, Aiii), can be seen to be spines. The TBS slice with potentiated synapses ( $B$ ii, Biii) has numerous green to yellow puncta, indicative of dense labeling;

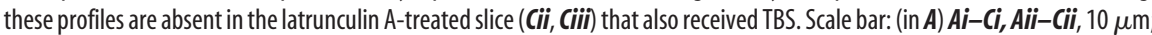
Aiii-Ciii, $5 \mu \mathrm{m}$. D, Plot describes spine frequency (expressed as a cumulative fraction of the total number of labeled spines) as a function of mean spine label intensity for groups of slices receiving baseline/control stimulation (CON, blue dashed line), TBS (red solid line), or TBS in the presence of $500 \mathrm{~nm}$ latrunculin A (green solid line). The intensity distribution for TBS-treated slices is significantly shifted toward high-intensity labeling compared with controls ( $K-S$ test, $D=0.25 ; p<0.0001$ ), whereas latrunculin A-treated slices exhibited almost no intensely labeled spines ( $K-S$ test vs controls, $D=0.10 ; p<0.01$ ). $E$, Bar graph plots group mean \pm SEM values for the total number of identifiable spines (with label intensity of $\geq 75$ PIU) from the independent experiments contributing to data plotted in $\boldsymbol{D}$ : the total number of labeled spines did not differ across treatment groups $(p>0.7$, one-way ANOVA). $\boldsymbol{F}$, Graph shows group mean \pm SEM values for numbers of intensely labeled spines (label intensity of $\geq 145$ PIU) from independent experiments contributing to data plotted in $\boldsymbol{D}$ : values demonstrate that infusion of latrunculin $A$ before TBS eliminated the presence of intensely labeled spines normally produced by $\operatorname{TBS}\left({ }^{* *} p<0.01\right.$, two-tailed $t$ test vs control group). $\mathbf{G}$, Graph shows fEPSP slopes recorded from control slices (aCSF, open circles) or those receiving bath application of $500 \mathrm{~nm}$ latrunculin A (filled circles) for 30 min beginning 20 min before TBS (arrow). In latrunculin A-treated slices, LTP was significantly attenuated by $2 \mathrm{~h}$ after TBS compared with aCSF controls ( $p<0.01$, two-way repeated-measures ANOVA for minutes $100-120$ ). theta stimulation reaches sufficient concentrations, and is appropriately located, to facilitate the formation of F-actin. The soluble TrkB-Ig construct TrkB-Fc sequesters and blocks the actions of BDNF (Shelton et al., 1995; Chen et al., 1999), presumably by preventing it from reaching its receptors. In agreement with this, pretreatment of adult hippocampal slices with TrkB-Fc for 20 min completely blocks Trk phosphorylation generated by applications of exogenous BDNF (Rex et al., 2006). The results summarized in Figure 6, $A$ and $B$, show that TrkB-Fc $(1 \mu \mathrm{g} /$ $\mathrm{ml}$ ) greatly reduced the number of densely phalloidin-labeled spine heads as assessed 20 min after delivery of a 10-burst theta train. Mean values for slices stimulated alone or in the presence of TrkB-Fc were $37 \pm 3$ and $11 \pm 4$, respectively $(p=$ 0.001 , two-tailed $t$ test). TrkB-Fc did not affect baseline F-actin levels, as evidenced by the absence of effect on spine label intensity distributions ( $\mathrm{K}-\mathrm{S}$ test, $D=0.02$; $p>0.05$ ) (Fig. 6C). To corroborate results obtained with TrkB-Fc, effects of the tyrosine and serine/threonine kinase inhibitor K252a (Schneider et al., 2005), which blocks signaling through the Trk neurotrophin receptors but not through p75 (Knusel and Hefti, 1992; Scharfman, 1997; Cosgaya et al., 2002; Cheng and Yeh, 2005; Skoff and Adler, 2006), were examined. K252a applied at $2 \mu \mathrm{M}$ does not alter basal synaptic transmission (Rex and Lynch, unpublished observations) and in the present studies did not affect the number of densely labeled spine profiles under baseline stimulation conditions $(9 \pm 3$ vs $11 \pm 4$ for control vs control $+\mathrm{K} 252 \mathrm{a}$, respectively; $p>0.4$, two-tailed $t$ test; $n=$ 3 per group). However, TBS-induced increases in densely labeled spine profiles were blocked by the inhibitor $(28 \pm 3$ vs $14 \pm 4$ for TBS vs TBS + K252a; $p<0.05$, two-tailed $t$ test; $n=5$ per group). This result is consistent with the conclusion that effects of TBS on actin polymerization depend on BDNF signaling through TrkB.

Endogenous BDNF could promote actin polymerization by either enhancing induction events (e.g., burst responses and NMDA receptor actions) initiated by TBS or acting on assembly processes that follow stimulation. TrkB-Fc does not measurably alter baseline synaptic transmission in hippocampus (Chen et al., 1999), and there was no evidence in the present study that the scavenger affected burst responses or the degree to which the bursts facilitate during a theta train (the increase in response area for bursts 2-10 relative to the first burst was $70 \pm 5 \%$ in controls and 
$66 \pm 5 \%$ in the TrkB-Fc group; $p>0.6$ ). To test whether the depressive effects of TrkB-Fc on F-actin labeling occurred after LTP induction, we delivered TrkB-Fc $(2 \mu \mathrm{g} / \mathrm{ml})$ directly upstream from the CA1 recording site via perfusion pipette for 2 min beginning $30 \mathrm{~s}$ after TBS. Figure $6 \mathrm{D}$ shows typical results: slices that received 2 min control $(\mathrm{IgG}-\mathrm{Fc})$ treatments had numerous densely labeled spines, whereas those infused with TrkB-Fc did not. Group results (Fig. 6E) confirmed that post-theta TrkB-Fc markedly reduced the number $(12 \pm 3)$ of densely labeled spines relative to that found in slices given control IgG-Fc infusions $(29 \pm 5 ; p<0.01$, two-tailed $t$ test). It appears, then, that assembly and/or maintenance of actin filaments in the immediate post-theta period requires the presence of endogenous BDNF.

BDNF activates pathways leading to actin polymerization

Work with dissociated cells indicates that BDNF regulates actin assembly in developing filopodia through Rho family GTPases that promote phosphorylation of actin depolymerizing factor (ADF)/cofilin (Fass et al., 2004; Gehler et al., 2004). There is also experimental evidence that BDNF influences growth in neuronal cultures through Rac (Yuan et al., 2003), a second Rho family member that acts via PAK and LIM (Lin-11/Isl-1/Mec-3) kinase to control cofilin activity (Carlisle and Kennedy, 2005). We tested whether these pathways are present in mature spines and responsive to BDNF at concentrations that promote actin polymerization. Cofilin is reported to be concentrated in spines within field CA1 of adult hippocampus (Racz and Weinberg, 2006). PAK proteins are known to be expressed by hippocampal neurons (Boda et al., 2004; Zhang et al., 2005), but information on their subcellular distributions has been restricted to immature systems (Meng et al., 2005). Figure $7 A$ shows double immunostaining for PAK3, a $\mathrm{PAK}$ isoform implicated in nonsyndromic X-linked mental retardation and spine morphogenesis (for review, see Allen et al., 1998; Boda et al., 2004) and PSD-95 in field CA1 of adult hippocampus. High-resolution images obtained by confocal microscopy revealed that the distribution of PAK3 immunostaining is remarkably discrete, with virtually all neuropil staining occurring as diffusely distributed, well labeled puncta, typically also immunoreactive for PSD-95 (Fig. 7B). In many of the double-labeled puncta, PAK3-IR extended beyond the boundaries of PSD-95-IR, indicating that PAK3 extends into the spine cytoplasm. PAK3 labeling was notably absent from dendritic shafts and axons, but perinuclear staining within the CA1 pyramidal cells was observed (Fig. 7C, left). The latter was similar to patterns of cytoplasmic F-actin labeling occasionally seen in phalloidin-incubated slices (Fig. 7C, right). Photomontages of the entire CA1 field assembled from high-magnification images were used for quantitative anal(initiated 20 min before recordings).
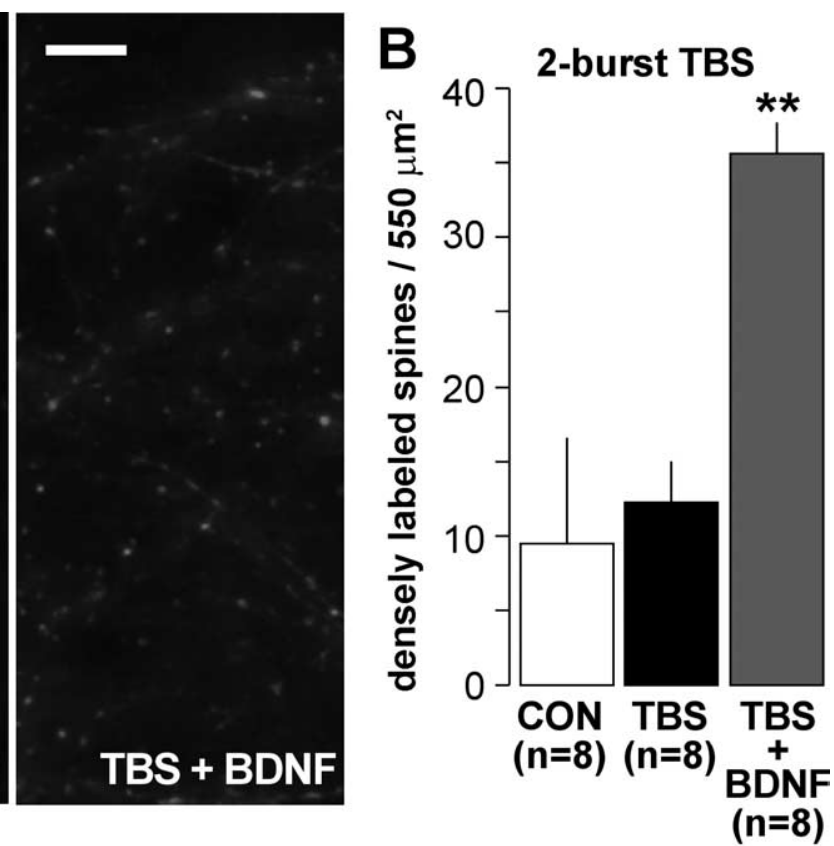

BDNF

0'

15

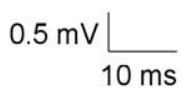

Figure 5. BDNF lowers the threshold for TBS-induced increases in spine F-actin. Hippocampal slices were treated with rholices receiving two theta bursts alone (TBS) had comparable numbers of F-actin dense spines as those receiving only baseline stimulation (CON: $p>0.3$ two-tailed $t$ test), whereas slices treated with two theta bursts in the presence of 2 nM BDNF (TBS + BDNF) had approximately threefold greater numbers of intensely labeled spines ( ${ }^{* *} p<0.01$ vs TBS alone). C, Field EPSPs recorded immediately before $\left(0^{\prime}\right)$ and 15 min after $\left(15^{\prime}\right)$ delivery of two theta bursts to slices receiving aCSF (CON) or BDNF infusion

ysis of the incidence of PAK3 and PSD-95 colocalization in immunostained puncta. The results indicate that a large proportion of the PSD-95-positive puncta ( $57 \pm 8 \% ; n=3)$ also contain detectable PAK3-IR. These points establish that hippocampal spine heads contain concentrations of PAK protein.

Having confirmed that BDNF-sensitive, actin-related proteins are concentrated in the heads of mature spines, we tested whether their phosphorylation state is influenced by brief treatments with the neurotrophin or its antagonist. BDNF was infused for $30 \mathrm{~min}$, after which hippocampal slices were processed for Western blot analysis. Pilot studies measuring levels of phosphorylated (p-) Trk determined that BDNF produces peak effects at $60 \mathrm{ng} / \mathrm{ml}$ in acute hippocampal slices. Figure $7 \mathrm{D}$ shows blots demonstrating that treatment with this concentration of BDNF increased levels of p-PAK (from $2785 \pm 52$ to $4573 \pm 761$ optical density units) and p-cofilin (from $2995 \pm 35$ to $4538 \pm 664$ ) by $>50 \%$ ( $p<0.05$ for both proteins). TrkB-Fc application beginning $15 \mathrm{~min}$ before and continuing throughout $30 \mathrm{~min}$ BDNF treatment blocked increases in both p-PAK ( $p>0.05$ vs controls for $0.2,1.0$, and $2.0 \mu \mathrm{g} / \mathrm{ml} \mathrm{TrkB-Fc}$ ) and p-cofilin ( $p>0.05 \mathrm{vs}$ controls for 1.0 and $2.0 \mu \mathrm{g} / \mathrm{ml}$ ) (Fig. $7 E$ ). Blots stripped and 

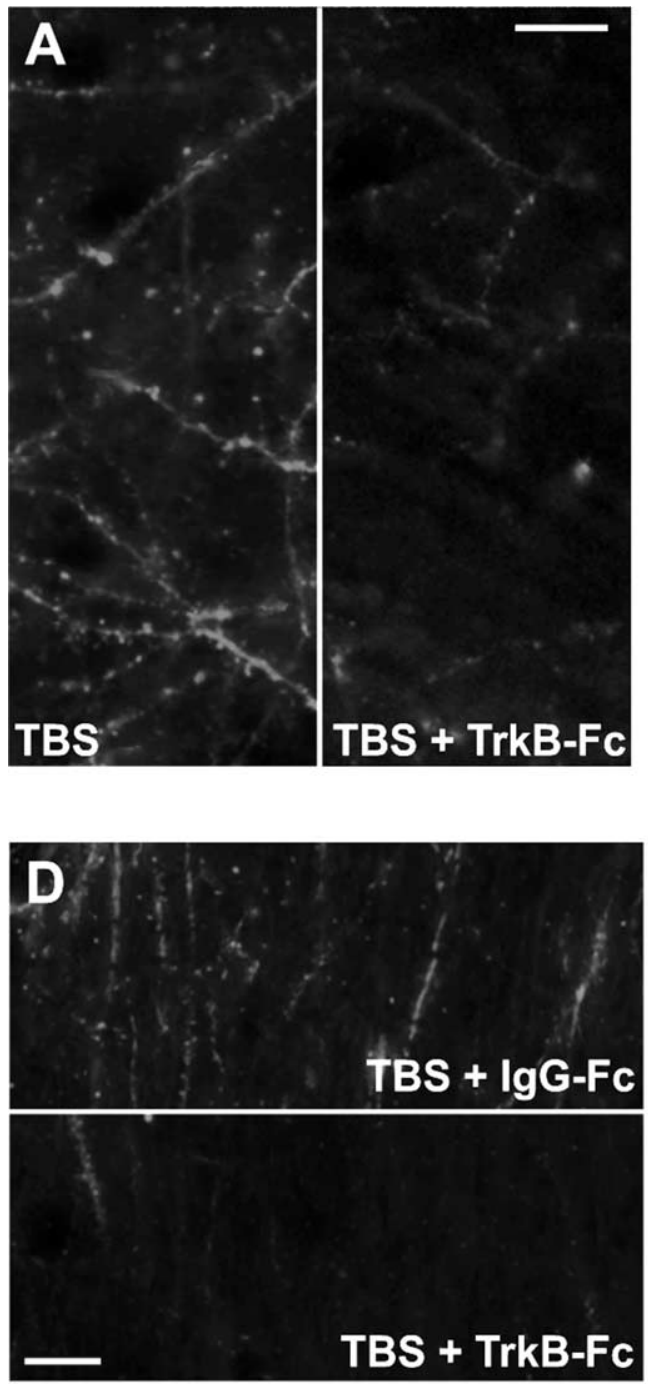
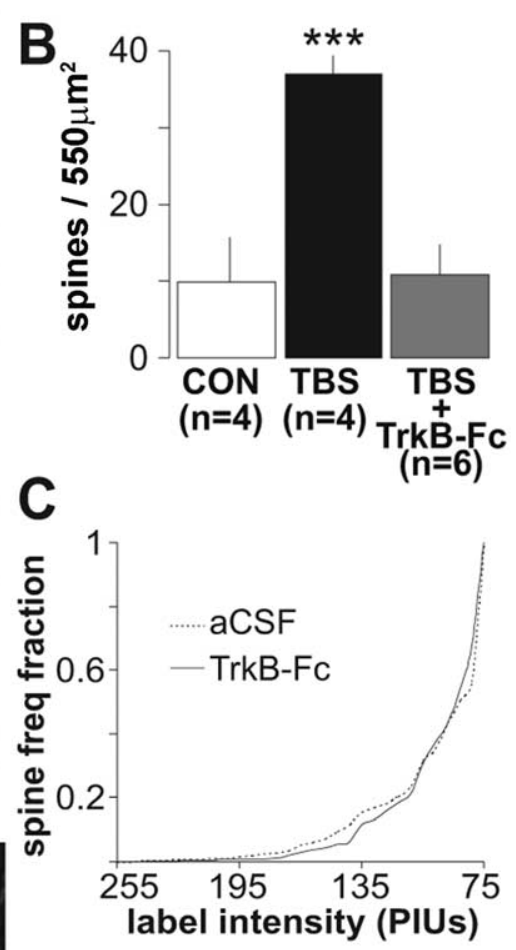

E

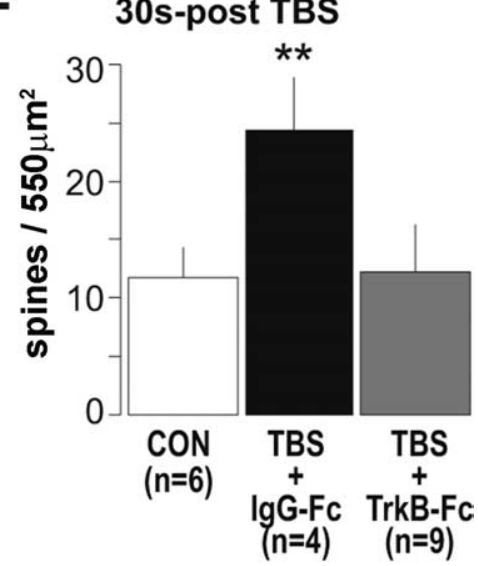

Figure 6. Sequestering extracellular BDNF prevents TBS-induced actin polymerization. $\boldsymbol{A}$, Representative photomicrographs of the CA1 sampling region shows a scattering of densely phalloidin-labeled spines in a slice given TBS alone and the absence of dense spine labeling in a slice given TBS in the presence of $1 \mu \mathrm{g} / \mathrm{ml}$ TrkB-Fc (TBS + TrkB-Fc; scale bar, $10 \mu \mathrm{m}$ ). B, A plot of group mean \pm SEM spine counts confirms that TBS markedly increased numbers of intensely labeled spines relative to controls (CON; ${ }^{* * *} p=0.001$, two-tailed $t$ test) and that this effect was blocked by TrkB-Fc ( $p>0.8$ vs controls). C, Cumulative fraction spine intensity plots (as in Fig. $3 H$ ) show similar intensity distributions for phalloidin-labeled spines for control slices maintained in aCSF (dashed line) and slices treated with $1 \mu \mathrm{g} / \mathrm{ml}$ TrkB-Fc alone (solid line; i.e., in the absence of TBS): no differences between TrkB-Fc and aCSF slices were detected ( $p>0.8$, two-way repeated-measures ANOVA). $\boldsymbol{D}$, Photomicrographs of rhodaminephalloidin labeling in field CA1 in slices receiving the control lg, lgG $-\mathrm{Fc}(\mathrm{TBS}+\mathrm{lgG}-\mathrm{Fc})$, or $2 \mu \mathrm{g} / \mathrm{ml} \operatorname{TrkB}-\mathrm{Fc}(\mathrm{TBS}+\mathrm{TrkB}-\mathrm{Fc})$ for 2 min starting $30 \mathrm{~s}$ after TBS. $\boldsymbol{E}$, Quantification of densely labeled spines shows that post-TBS application of $\mathrm{lgG}-\mathrm{Fc}$ did not block theta-induced increases in the number of intensely labeled spines ( ${ }^{* *} p<0.01$ vs control, two-tailed $t$ test), whereas post-TBS infusion of TrkB-Fc eliminated the effect.

reprobed for total protein revealed no effects of treatment on total PAK3 or cofilin (Fig. 7D). These results suggest that elevated levels of released BDNF, as occur with TBS (Aicardi et al., 2004), are likely to exert an acute influence over the status of proteins regulating actin assembly. As described above, BDNF applied alone does not initiate actin polymerization in adult slices. In accord with this, TrkB-Fc applied at concentrations that completely blocked BDNF-induced increases in p-Trk did not alter baseline levels of p-PAK or p-cofilin in hippocampal slices $(p>0.3$, one-way ANOVA). It appears, then, that background levels of BDNF release do not tonically affect the Rac-PAK-cofilin-actin pathway.

\section{Effects of a BDNF antagonist on LTP consolidation}

Evidence on whether BDNF promotes LTP consolidation in the minutes after induction (as predicted by the above results) is mixed. BDNF applied during, but not after, stimulation facilitates LTP in the dentate gyrus (Kovalchuk et al., 2002) and BDNF function-blocking antiserum, infused during and immediately after TBS, blocks LTP stabilization (Kossel et al., 2001). Importantly, in the latter studies, the BDNF antiserum was ineffective when applied 10 min later. In contrast, Kang et al. (1997) reported that TrkB-Fc reverses TBS-induced LTP when applied $30 \mathrm{~min}$ after induction, suggesting that BDNF is needed for the maintenance of potentiation. Given these discrepancies, and the absence of direct tests of endogenous BDNF effects on LTP consolidation in the immediate post-induction period, we reexamined the effects of TrkB-Fc, at concentrations that block TBS-induced phalloidin staining, on the formation of stable LTP.

As shown in Figure $8 B$, a 2 min local infusion of TrkB-Fc, applied $30 \mathrm{~s}$ after TBS, caused potentiation to decay steadily until responses reached a plateau that was significantly lower (116 $\pm 8 \%$ of baseline fEPSP slope) than potentiation induced in a second pathway $(142 \pm 5 \%$ at $30 \mathrm{~min}$ after TBS) after a 28 min washout of the scavenger ( $p<0.05$, two-way repeatedmeasures ANOVA for 20-30 min after TBS). Figure $8 C$ describes experiments in which TrkB-Fc was replaced with the control IgG-Fc: potentiation stabilized at levels $(137 \pm 6 \%)$ similar to the control pathway (above) $(p<0.05$ for IgG-Fc vs TrkB-Fc applied $30 \mathrm{~s}$ after TBS). Because TrkB-Fc was applied after induction and initial expression of potentiation, it follows that it affected LTP by disrupting consolidation. To further test this point, we applied TrkB-Fc at $10 \mathrm{~min}$ after TBS (Fig. $8 D$ ), a time point at which potentiation is considerably more resistant to disruption (Arai et al., 1990; Huang et al., 1999) and increases in extracellular BDNF triggered by theta stimulation begin to dissipate (Aicardi et al., 2004). The group results summarized in Figure $8 E$ show that TrkB-Fc did not detectably alter potentiation when applied at this time point, indicating that its actions on synaptic plasticity are time dependent. We confirmed this in a separate synaptic population (str. oriens) on the same group of neurons (Fig. $8 E$ ). 

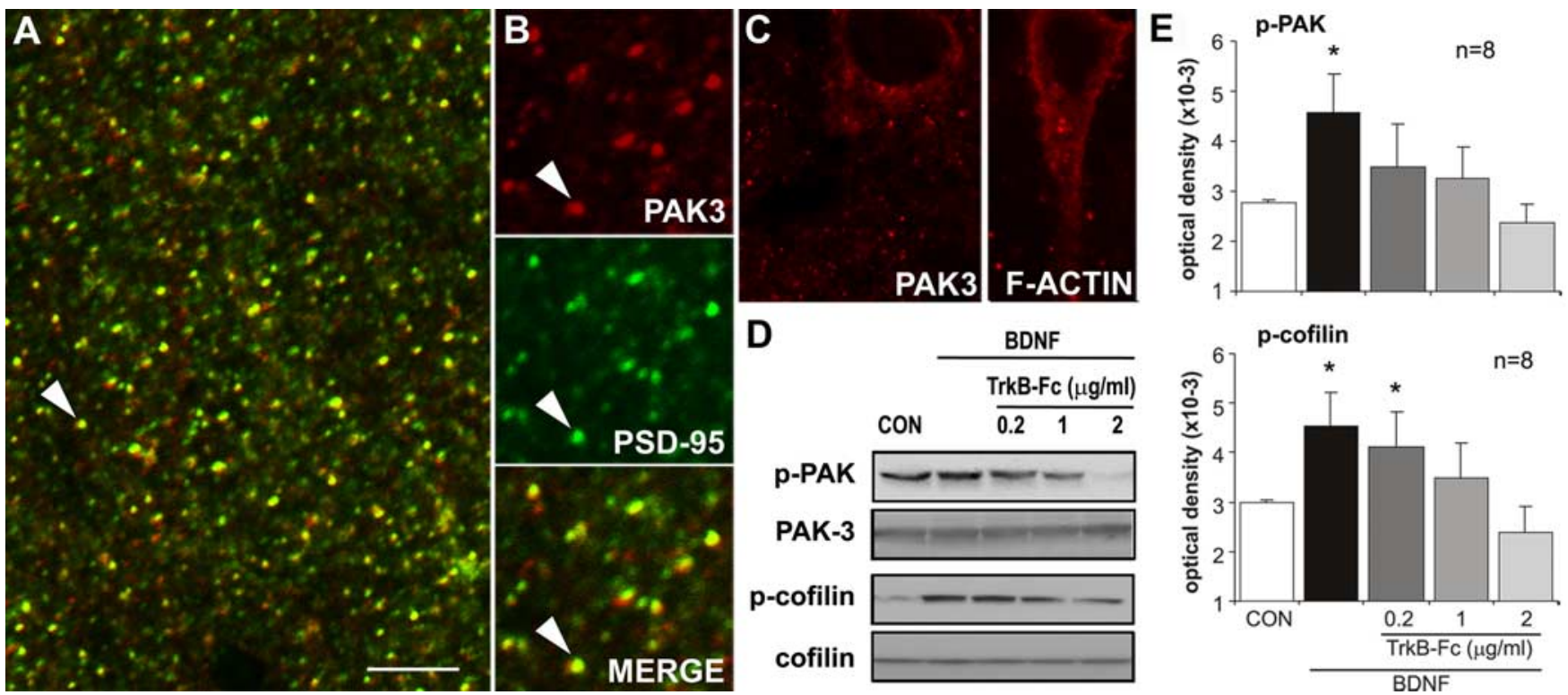

Figure 7. Exogenous BDNF promotes phosphorylation of actin regulatory proteins. A, High-power confocal micrograph shows anti-PSD-95 (green) and anti-PAK3 (red) double labeling in field CA1 (double-labeled elements appearyellow). Both markers labeled discrete puncta scattered throughout str. radiatum. Scale bar: $\boldsymbol{A}, 5 \mu \mathrm{m} ; \boldsymbol{B}, 3 \mu \mathrm{m} . \boldsymbol{B}$, Enlargements of the field including the arrow in $\boldsymbol{A}$ separated into red (PAK3), green (PSD-95), and overlaid (Merge) channels ( $\boldsymbol{A}$ is a "merged" image) show that most, but not all, PAK3-immunoreactive puncta also contain PSD-95-IR. $\boldsymbol{C}$, Micrographs show perinuclear localization of PAK3-IR (left) and phalloidin-labeled F-actin (right). $\boldsymbol{D}, \boldsymbol{E}$, Adult rat hippocampal slices were treated with BDNF (60 ng/ml; bath applied) for $30 \mathrm{~min}$ alone or with TrkB-Fc pretreatment. Representative Western blots (D) show treatment effects on p-PAK immunoreactivity (detects the conserved phosphorylation site on p-PAK isoforms 1, 2, and 3) with no evident changes in total PAK3. BDNF-induced increases in p-PAK were eliminated by TrkB-Fc in a dose-dependent manner. BDNF similarly increased, and TrkB-Fc similarly reduced, levels of p-cofilin without effects on total cofilin content. $\boldsymbol{E}$, Quantification of Western blots assessing BDNF treatment effects on p-PAK (top) and p-cofilin (bottom) immunoreactivities, with and without TrkB-Fc cotreatment (group mean \pm SEM band densities shown; $n=8$ per group). In each case, BDNF-induced increases in the phosphoproteins $\left({ }^{*} p<0.05\right.$ vs control, one-way ANOVA followed by Tukey's HSD test) were blocked by TrkB-Fc at concentrations from $0.2-2 \mu \mathrm{g} / \mathrm{ml}$ for $\mathrm{p}-\mathrm{PAK}$ and $1-2 \mu \mathrm{g} / \mathrm{ml}$ for $\mathrm{p}$-cofilin.

\section{Discussion}

The present results establish that the effects of BDNF on the developing neuronal cytoskeleton persist, in a somewhat muted form, into adulthood. The neurotrophin caused rapid phosphorylation, and hence inactivation, of $\mathrm{ADF} / \mathrm{cofilin}$, a protein located in spine heads and known to cleave actin filaments. It similarly increased levels of phosphorylated PAK, a Rac effector that we found to be greatly concentrated in adult spines and that, through LIM kinase, controls cofilin phosphorylation (Carlisle and Kennedy, 2005). Presumably related to its actions on PAK and cofilin, BDNF markedly enhanced the number of dense, punctate concentrations of F-actin produced by otherwise subthreshold levels of theta burst stimulation. We confirmed previous suggestions (Lin et al., 2005a; Kramar et al., 2006) that these puncta are spine heads by showing that they colocalize with the postsynaptic density protein PSD-95 and that they represent newly polymerized actin by establishing that their occurrence is prevented by latrunculin A. Together, these results suggested that endogenous BDNF, which is released by TBS, promotes the cytoskeletal changes that accompany and support LTP. As predicted by this idea, the BDNF scavenger TrkB-Fc prevented the actin polymerization produced by suprathreshold levels of theta stimulation; similar results were obtained with the Trk signaling antagonist K252a. Effects of TrkB-Fc were not secondary to any actions of the scavenger on the theta burst responses that trigger polymerization because it occurred with TrkB-Fc applications that began $30 \mathrm{~s}$ after theta stimulation. In all, then, it is likely that released BDNF acts on Rho-dependent pathways to directly promote the assembly of actin filaments in spine heads during the first few minutes after the induction of LTP. Importantly, BDNF by itself did not increase F-actin levels, indicating that its potency or range of action is reduced from what is found during development.
The above conclusions provide an explanation for the positive effects of BDNF on the production of stable LTP (Figurov et al., 1996; Kang et al., 1997). Multiple lines of evidence indicate that theta-induced actin polymerization is critical for consolidation: the effect has the same threshold as LTP, is reversed by manipulations that reverse LTP, and becomes increasingly stable over the same 30 min time period that covers the rapid phase of LTP consolidation (Kramar et al., 2006). Moreover, treatments that block F-actin assembly block LTP (Krucker et al., 2000). Given these results, BDNF, as the first reported positive modulator of activity-induced actin polymerization, has predictable effects on LTP. It remains possible that it also acts on other aspects of the potentiation effect. Infused BDNF enhances transmitter release during high-frequency stimulation (Gottschalk et al., 1998) and depresses the afterhyperpolarizations that accompany theta trains (Kramar et al., 2004), two actions that should have strongly positive effects on LTP induction. However, we did not detect any actions of TrkB-Fc and thus of endogenous BDNF signaling through TrkB, on high-frequency theta burst responses or their facilitation during a theta train. This could indicate that the neurotrophin is released late in the train, or even in the seconds following, and thus is not available to produce the changes in theta responses seen with exogenous BDNF infusion. It will be of interest in this regard to test whether a conventional theta burst train produces BDNF-like effects on responses to subsequent trains of theta stimulation.

The present results also establish that the post-induction effects of BDNF on LTP are time dependent. Infusions of the BDNF scavenger immediately after induction caused potentiation to decay but had no effect when begun after a 10 min delay. There is disagreement in the literature about whether disrupting the actions of extracellular BDNF at 10-30 min after theta stimulation 
A

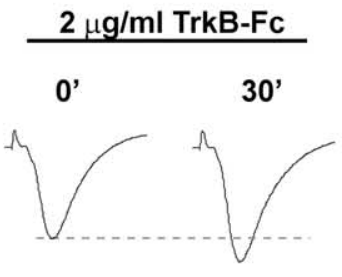

$\underset{10 \mathrm{~ms}}{\mathrm{~J}} 0.5 \mathrm{mV}$
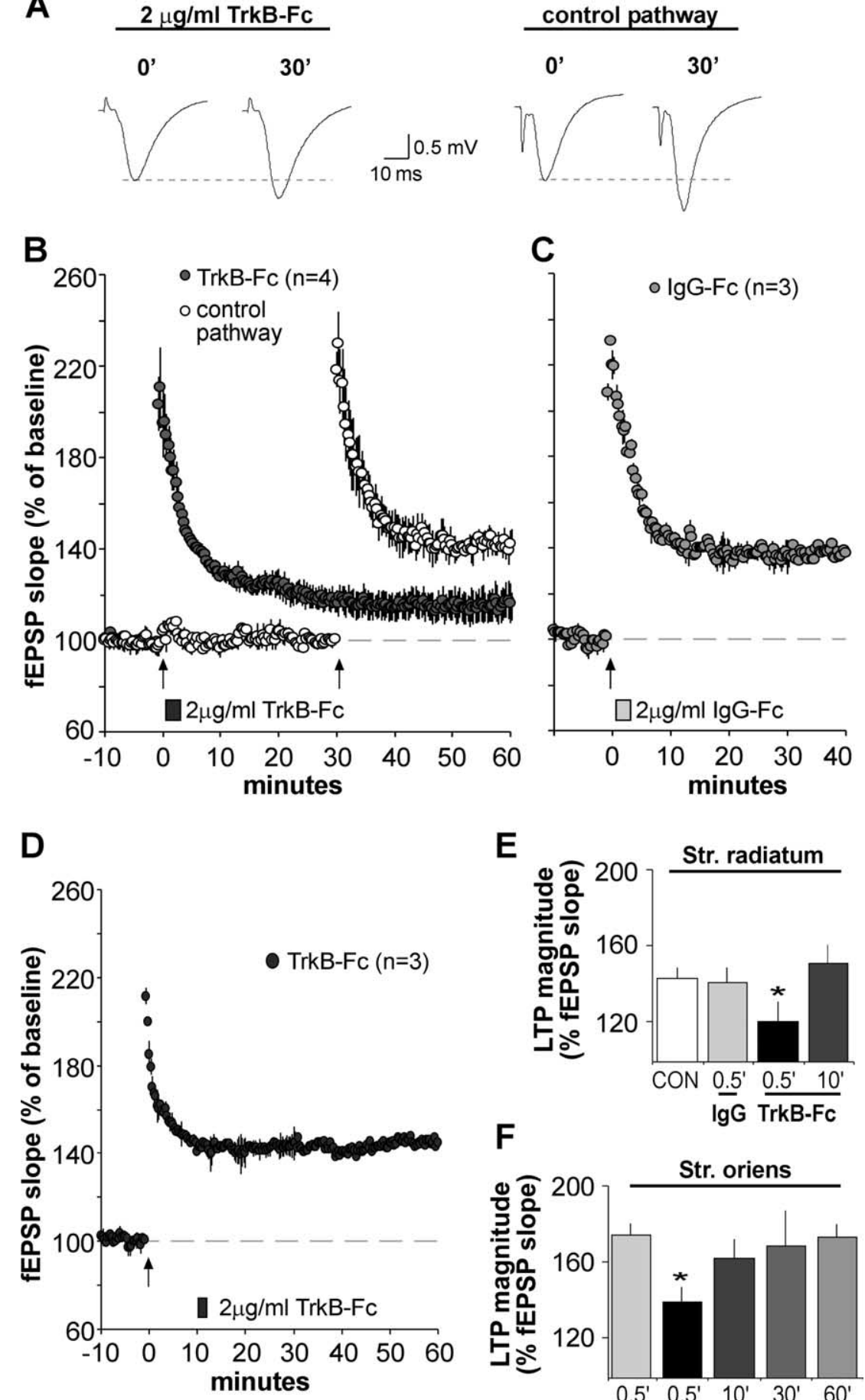

E
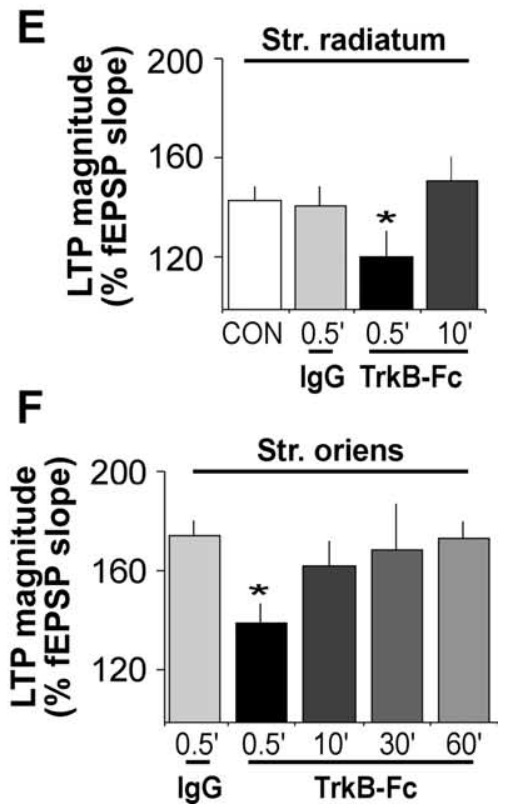

Figure 8. BDNF scavenger TrkB-Fc applied after theta burst stimulation disrupts LTP consolidation. $\boldsymbol{A}$, Representative fEPSP traces show that the degree of synaptic potentiation produced by a conventional theta burst train was attenuated when TrkB-Fc $(2 \mu \mathrm{g} / \mathrm{ml})$ was applied for $2 \mathrm{~min}$ beginning $30 \mathrm{~s}$ after TBS. Traces at right ( $2 \mu \mathrm{g} / \mathrm{ml}$ TrkB-Fc) were collected during baseline $\left(0^{\prime}\right)$ and 30 min after TBS $\left(30^{\prime}\right)$. The experiment was repeated using a second population of synapses (control pathway; traces at left) after the scavenger had been washed out for $27 \mathrm{~min}$. In this case, normal TBS-induced potentiation (30min after TBS) was obtained. $\boldsymbol{B}$, Plot shows group fEPSP slopes (means \pm SEM) for experiments described above. Note that potentiation for the pathway receiving post-TBS TrkB-Fc treatment (filled circles), although initially robust, decayed significantly compared with potentiation in the control pathway (open circles); arrow here and in later panels indicates placement of TBS. C, Same as in B except that the control lgG $-\mathrm{Fc}(2 \mu \mathrm{g} / \mathrm{ml})$ was infused 30 s after TBS; this had no effect on LTP. D, TrkB-Fc infused for 2 min starting $10 \mathrm{~min}$ after TBS had no effect on LTP. E, $F$, Summary of the percentage of LTP obtained with post-induction infusion of TrkB-Fc or IgG-FC: values are percentage change from baseline measured $30 \mathrm{~min}$ after infusion onset; tests performed in apical (str. radiatum) and basal (str. oriens) dendrites are summarized in $\boldsymbol{E}$ and $\boldsymbol{F}$, respectively. For both lamina, TrkB-Fc attenuated LTP expression when applied $30 \mathrm{~s}$ after TBS compared with potentiation attained in IgG-Fc-treated controls $\left(^{*} p<0.05\right.$, two-way repeated-measures ANOVA followed by Tukey's HSD) but failed to alter potentiation at later time points. does or does not affect LTP (Kang et al., 1997; Kossel et al., 2001). Our results accord with the idea that BDNF supports a memory-like consolidation process that begins within seconds of LTP induction and is well advanced $15-30$ min later. Evidence for such a process comes from experiments showing that diverse manipulations disrupt LTP when applied shortly after induction but become progressively less effective over the following $30 \mathrm{~min}$ (Barrionuevo et al., 1980; Arai et al., 1990; Staubli and Lynch, 1990; Huang et al., 1999). That TrkB-Fc was without any effect when applied 10 min after TBS suggests that BDNF works on early steps in the consolidation process; this is consonant with its actions on activity-induced actin polymerization, which is evident within 2 min after stimulation (Lin et al., 2005a). As noted, the assembled actin filaments become resistant to disruption (depolymerization) over the same period that LTP does (Kramar et al., 2006). Whether BDNF contributes to this is not addressed by the present studies, although the lack of any effect of TrkB-Fc at $10 \mathrm{~min}$ after induction suggests that the role of BDNF is complete, whereas capping, crosslinking, and other steps that stabilize the actin filaments are still in progress. Our results also leave open the possibility that BDNF contributes to later phases of consolidation involving protein synthesis (Frey and Morris, 1997; Smart et al., 2003; Pang and $\mathrm{Lu}, 2004$ ).

Collectively, the above findings raise questions about the nature of (1) theta burst-driven signaling that BDNF enhances so as to produce actin polymerization and (2) the developmental changes that seemingly constrain the influence of neurotrophin over the cytoskeleton in the adult. Experiments using various ligands (Xiao et al., 1991; Bahr et al., 1997), disintegrins (Chun et al., 2001), neutralizing antibodies (Chun et al., 2001; Kramár et al., 2002; Kramar and Lynch, 2003), and mutant mice (Chan et al., 2006) indicate that cell adhesion receptors belonging to the $\beta 1$ integrin family play a critical role in LTP consolidation. This is of particular interest in the present context because integrins regulate the actin cytoskeleton in tissues throughout the body (Wiesner et al., 2005) and thus are logical candidates for the agency that initiates actin changes that follow theta burst stimulation. This argument was confirmed in experiments showing that neutralizing antibodies against $\beta 1$ integrins completely suppress both theta-induced increases in $\mathrm{F}$-actin and LTP consolidation (Kramar et al., 
2006). Interactions between integrin and trophic factor receptor signaling cascades are commonplace (Fu et al., 2001; Miranti and Brugge, 2002; Yamada and Even-Ram, 2002), and many trophic functions are now understood to require, or to be enhanced by, the engagement of both classes of receptors (ffrench-Constant and Colognato, 2004; Tucker et al., 2005). It is thus reasonable to anticipate that integrin- and BDNF-mediated signaling cascades set in motion by theta stimulation converge on downstream elements regulating actin polymerization. The present results support this possibility. Integrin and growth factor signaling are known to converge on Rac (Le et al., 2005) and its effector PAK (Slack-Davis et al., 2003). As reported here, BDNF stimulates increases in PAK phosphorylation. Future studies will be necessary to determine whether these changes are confined to synapses, but studies in progress have demonstrated increases in p-PAK immunoreactivity in adult rat forebrain synaptoneurosomes after treatment with integrin ligands (our unpublished observations). This convergence of signaling suggests that subthreshold activation of integrins could be boosted to a functionally effective range by the neurotrophin (and visa versa). The BDNF effects reported here would thus represent a special case of a widely distributed type of receptor synergy.

The integrin/BDNF interaction hypothesis may also help explain why, in adult brain, the neurotrophin alone does not initiate the rapid structural changes seen in immature neurons. Integrins, because of their influence over the cytoskeleton and growth-related genes, contribute critically to cell maturation (Juliano, 1998; Streuli and Edwards, 1998). Certain of the adhesion receptors arrive in hippocampal dendrites (Bi et al., 2001) and come to influence synaptic physiology (Kramar and Lynch, 2003) relatively late in postnatal development, at a time point at which synaptogenesis begins to slow. Possibly, then, among its other suppressive effects, integrin signaling exerts a tonic negative control over TrkB receptors and their associated signaling pathways. Combining these points, we can postulate that latearriving integrins block or constrain the TrkB-to-actin signaling and that this condition is transiently relaxed, or converted to a positive interaction, by theta pattern activity. Thus, a pattern of cortical activity associated with learning would both release BDNF from terminals and unblock its receptor.

\section{References}

Ackermann M, Matus A (2003) Activity-induced targeting of profilin and stabilization of dendritic spine morphology. Nat Neurosci 6:1194-1200.

Aicardi G, Argilli E, Cappello S, Santi S, Riccio M, Thoenen H, Canossa M (2004) Induction of long-term potentiation and depression is reflected by corresponding changes in secretion of endogenous brain-derived neurotrophic factor. Proc Natl Acad Sci USA 101:15788-15792.

Allen KM, Gleeson JG, Bagrodia S, Partington MW, MacMillan JC, Cerione RA, Mulley JC, Walsh CA (1998) PAK3 mutation in nonsyndromic X-linked mental retardation. Nat Genet 20:25-30.

Arai A, Larson J, Lynch G (1990) Anoxia reveals a vulnerable period in the development of long-term potentiation. Brain Res 511:353-357.

Arai A, Black J, Lynch G (1994) Origins of the variations in long-term potentiation between synapses in the basal versus apical dendrites of hippocampal neurons. Hippocampus 4:1-9.

Bahr BA, Staubli U, Xiao P, Chun D, Ji Z-X, Esteban ET, Lynch G (1997) Arg-Gly-Asp-Ser-selective adhesion and the stabilization of long-term potentiation: pharmacological studies and the characterization of a candidate matrix receptor. J Neurosci 17:1320-1329.

Balkowiec A, Katz DM (2002) Cellular mechanisms regulating activitydependent release of native brain-derived neurotrophic factor from hippocampal neurons. J Neurosci 22:10399-10407.

Barde YA (1994) Neurotrophins: a family of proteins supporting the survival of neurons. Prog Clin Biol Res 390:45-56.

Barrionuevo G, Schottler F, Lynch G (1980) The effects of repetitive low frequency stimulation on control and "potentiated" synaptic responses in the hippocampus. Life Sci 27:2385-2391.

Bernstein BW, DeWit M, Bamburg JR (1998) Actin disassembles reversibly during electrically induced recycling of synaptic vesicles in cultured neurons. Brain Res Mol Brain Res 53:236-251.

Bi X, Lynch G, Zhou J, Gall CM (2001) Polarized distribution of $\alpha 5$ integrin in the dendrites of hippocampal and cortical neurons. J Comp Neurol 435:184-193.

Boda B, Alberi S, Nikonenko I, Node-Langlois R, Jourdain P, Moosmayer M, Parisi-Jourdain L, Muller D (2004) The mental retardation protein PAK3 contributes to synapse formation and plasticity in hippocampus. J Neurosci 24:10816-10825.

Carlisle HJ, Kennedy MB (2005) Spine architecture and synaptic plasticity. Trends Neurosci 28:182-187.

Chan CS, Weeber EJ, Zong L, Fuchs E, Sweatt JD, Davis RL (2006) $\beta 1$ Integrins are required for hippocampal AMPA receptor-dependent synaptic transmission, synaptic plasticity, and working memory. J Neurosci 26:223-232.

Chen G, Kolbeck R, Barde YA, Bonhoeffer T, Kossel A (1999) Relative contribution of endogenous neurotrophins in hippocampal long-term potentiation. J Neurosci 19:7983-7990.

Cheng Q, Yeh HH (2005) PLCgamma signaling underlies BDNF potentiation of Purkinje cell responses to GABA. J Neurosci Res 79:616-627.

Chun D, Gall CM, Bi X, Lynch G (2001) Evidence that integrins contribute to multiple stages in the consolidation of long-term potentiation. Neuroscience 105:815-829.

Cooper JA (1987) Effects of cytochalasin and phalloidin on actin. J Cell Biol 105:1473-1478.

Cosgaya JM, Chan JR, Shooter EM (2002) The neurotrophin receptor p75NTR as a positive modulator of myelination. Science 298:1245-1248.

Fass J, Gehler S, Sarmiere P, Letourneau P, Bamburg JR (2004) Regulating filopodial dynamics through actin-depolymerizing factor/cofilin. Anat Sci Int 79:173-183.

ffrench-Constant C, Colognato H (2004) Integrins: versatile integrators of extracellular signals. Trends Cell Biol 14:678-686.

Figurov A, Pozzo-Miller LD, Olafsson P, Wang T, Lu B (1996) Regulation of synaptic responses to high-frequency stimulation and LTP by neurotrophins in the hippocampus. Nature 381:706-709.

Fischer M, Kaech S, Knutti D, Matus A (1998) Rapid actin-based plasticity in dendritic spines. Neuron 20:847-854.

Frerking M, Malenka RC, Nicoll RA (1998) Brain-derived neurotrophic factor (BDNF) modulates inhibitory, but not excitatory, transmission in the CA1 region of the hippocampus. J Neurophysiol 80:3383-3386.

Frey U, Morris RG (1997) Synaptic tagging and long-term potentiation. Nature 385:533-536.

Fu WM, Liou HH, Wang CL (2001) Collaboration of fibronectin matrix and neurotrophin in regulating spontaneous transmitter release at developing neuromuscular synapses in Xenopus cell cultures. Neurosci Lett 300:115-119.

Fukazawa Y, Saitoh Y, Ozawa F, Ohta Y, Mizuno K, Inokuchi K (2003) Hippocampal LTP is accompanied by enhanced F-actin content within the dendritic spine that is essential for late LTP maintenance in vivo. Neuron 38:447-460.

Geeraert V, Dupont JL, Grant NJ, Huvet C, Chasserot-Golaz S, Janoshazi A, Procksch O, de Barry J (2003) F-actin does not modulate the initial steps of the protein kinase $\mathrm{C}$ activation process in living nerve cells. Exp Cell Res 289:222-236.

Gehler S, Shaw AE, Sarmiere PD, Bamburg JR, Letourneau PC (2004) Brain-derived neurotrophic factor regulation of retinal growth cone filopodial dynamics is mediated through actin depolymerizing factor/ cofilin. J Neurosci 24:10741-10749.

Gottschalk W, Pozzo-Miller LD, Figurov A, Lu B (1998) Presynaptic mod ulation of synaptic transmission and plasticity by brain-derived neurotrophic factor in the developing hippocampus. J Neurosci 18:6830-6839.

Huang CC, Liang YC, Hsu KS (1999) A role for extracellular adenosine in time-dependent reversal of long-term potentiation by low-frequency stimulation at hippocampal CA1 synapses. J Neurosci 19:9728-9738.

Huang EJ, Reichardt LF (2001) Neurotrophins: roles in neuronal development and function. Annu Rev Neurosci 24:677-736.

Hunt CA, Schenker LJ, Kennedy MB (1996) PSD-95 is associated with the postsynaptic density and not with the presynaptic membrane at forebrain synapses. J Neurosci 16:1380-1388. 
Jain AK (1984) Fundamentals of digital image processing. Englewood Cliffs, NJ: Prentice Hall.

Juliano R (1998) Cooperation between soluable factors and integrinmediated cell anchorage in the control of cell growth and differentiation. BioEssays 18:911-917.

Kang H, Welcher AA, Shelton D, Schuman EM (1997) Neurotrophins and time: different roles for TrkB signaling in hippocampal long-term potentiation. Neuron 19:653-664.

Kim CH, Lisman JE (1999) A role of actin filament in synaptic transmission and long-term potentiation. J Neurosci 19:4314-4324.

Knusel B, Hefti F (1992) K-252 compounds: modulators of neurotrophin signal transduction. J Neurochem 59:1987-1996.

Kossel AH, Cambridge SB, Wagner U, Bonhoeffer T (2001) A caged Ab reveals an immediate/instructive effect of BDNF during hippocampal synaptic potentiation. Proc Natl Acad Sci USA 98:14702-14707.

Kovalchuk Y, Hanse E, Kafitz KW, Konnerth A (2002) Postsynaptic induction of BDNF-mediated long-term potentiation. Science 295:1729-1734.

Kramar EA, Lynch G (2003) Developmental and regional differences in the consolidation of long-term potentiation. Neuroscience 118:387-398.

Kramar EA, Bernard JA, Gall CM, Lynch G (2002) Alpha3 integrin receptors contribute to the consolidation of long-term potentiation. Neuroscience 110:29-39.

Kramar EA, Lin B, Lin CY, Arai AC, Gall CM, Lynch G (2004) A novel mechanism for the facilitation of theta-induced long-term potentiation by brain-derived neurotrophic factor. J Neurosci 24:5151-5161.

Kramar EA, Lin B, Rex CS, Gall CM, Lynch G (2006) Integrin-driven actin polymerization consolidates long-term potentiation. Proc Natl Acad Sci USA 103:5579-5584.

Krucker T, Siggins GR, Halpain S (2000) Dynamic actin filaments are required for stable long-term potentiation (LTP) in area CAl of the hippocampus. Proc Natl Acad Sci USA 97:6856-6861.

Lang C, Barco A, Zablow L, Kandel ER, Siegelbaum SA, Zakharenko SS (2004) Transient expansion of synaptically connected dendritic spines upon induction of hippocampal long-term potentiation. Proc Natl Acad Sci USA 101:16665-16670.

Larson J, Wong D, Lynch G (1986) Patterned stimulation at the theta frequency is optimal for the induction of hippocampal long-term potentiation. Brain Res 368:347-350.

Le SS, Loucks FA, Udo H, Richardson-burns S, Phelps RA, Bouchard RJ, Barth H, Aktories K, Tyler KL, Kandel ER, Heidenreich KA, Linseman DA (2005) Inhibition of Rac GTAase triggers a c-Jun- and Bim-dependent mitochondrial apoptotic cascade in cerebellar granule neurons. J Neurochem 94:1025-1039.

Lin B, Kramar EA, Bi X, Brucher FA, Gall CM, Lynch G (2005a) Theta stimulation polymerizes actin in dendritic spines of hippocampus. J Neurosci 25:2062-2069.

Lin CY, Lynch G, Gall CM (2005b) AMPA receptor stimulation increases alpha5betal integrin surface expression, adhesive function and signaling. J Neurochem 94:531-546.

Lu B, Pang PT, Woo NH (2005) The yin and yang of neurotrophin action. Nat Rev Neurosci 6:603-614.

Matus A (2000) Actin-based plasticity in dendritic spines. Science 290:754-758.

Meng J, Meng Y, Hanna A, Janus C, Jia Z (2005) Abnormal long-lasting synaptic plasticity and cognition in mice lacking the mental retardation gene Pak3. J Neurosci 25:6641-6650.

Miller FD, Kaplan DR (2001) On Trk for retrograde signaling. Neuron 32:767-770.

Miller FD, Kaplan DR (2003) Signaling mechanisms underlying dendrite formation. Curr Opin Neurobiol 13:391-398.

Miranti CK, Brugge JS (2002) Sensing the environment: a historical perspective on integrin signal transduction. Nat Cell Biol 4:83-90.

Nagerl UV, Eberhorn N, Cambridge SB, Bonhoeffer T (2004) Bidirectional activity-dependent morphological plasticity in hippocampal neurons. Neuron 44:759-767.
Okamoto K, Nagai T, Miyawaki A, Hayashi Y (2004) Rapid and persistent modulation of actin dynamics regulates postsynaptic reorganization underlying bidirectional plasticity. Nat Neurosci 7:1104-1112.

Pang PT, Lu B (2004) Regulation of late-phase LTP and long-term memory in normal and aging hippocampus: role of secreted proteins tPA and BDNF. Ageing Res Rev 3:407-430.

Racz B, Weinberg RJ (2006) Spatial organization of cofilin in dendritic spines. Neuroscience 138:447-456.

Rex CS, Kramar EA, Colgin LL, Lin B, Gall CM, Lynch G (2005) Long-term potentiation is impaired in middle-aged rats: regional specificity and reversal by adenosine receptor antagonists. J Neurosci 25:5956-5966.

Rex CS, Lauterborn JC, Lin CY, Kramar EA, Rogers GA, Gall CM, Lynch G (2006) Restoration of long-term potentiation in middle-aged hippocampus after induction of brain-derived neurotrophic factor. J Neurophysiol 96:677-685.

Scharfman HE (1997) Hyperexcitability in combined entorhinal/hippocampal slices of adult rat after exposure to brain-derived neurotrophic factor. J Neurophysiol 78:1082-1095.

Schneider TL, Mathew RS, Rice KP, Tamaki K, Wood JL, Schepartz A (2005) Increasing the kinase specificity of k252a by protein surface recognition. Org Lett 7:1695-1698.

Shelton DL, Sutherland J, Gripp J, Camerato T, Armanini MP, Phillips HS, Carroll K, Spencer SD, Levinson AD (1995) Human trks: molecular cloning, tissue distribution, and expression of extracellular domain immunoadhesins. J Neurosci 15:477-491.

Shimono K, Kubota D, Brucher F, Taketani M, Lynch G (2002) Asymmetrical distribution of the Schaffer projections within the apical dendrites of hippocampal field CA1. Brain Res 950:279-287.

Skoff AM, Adler JE (2006) Nerve growth factor regulates substance P in adult sensory neurons through both TrkA and p75 receptors. Exp Neurol 197:430-436.

Slack-Davis JK, Eblen ST, Zecevic M, Boerner SA, Tarcsafalvi A, Diaz HB, Marshall MS, Weber MJ, Parsons JT, Catling AD (2003) PAK1 phosphorylation of MEK1 regulates fibronectin-stimulated MAPK activation. J Biol Chem 162:281-291.

Smart FM, Edelman GM, Vanderklish PW (2003) BDNF induces translocation of initiation factor $4 \mathrm{E}$ to $\mathrm{mRNA}$ granules: evidence for a role of synaptic microfilaments and integrins. Proc Natl Acad Sci USA 100:14403-14408.

Staubli U, Lynch G (1990) Stable depression of potentiated synaptic responses in the hippocampus with $1-5 \mathrm{~Hz}$ stimulation. Brain Res 513:113-118.

Streuli CH, Edwards GM (1998) Control of normal mammary epithelial phenotype by integrins. J Mammary Gland Biol Neoplasia 3:151-163.

Swedlow JR, Hu K, Andrews PD, Roos DS, Murray JM (2002) Measuring tubulin content in Toxoplasma gondii: a comparison of laser-scanning confocal and wide-field fluorescence microscopy. Proc Natl Acad Sci USA 99:2014-2019.

Tucker BA, Rahimtula M, Mearow KM (2005) Integrin activation and neurotrophin signaling cooperate to enhance neurite outgrowth in sensory neurons. J Comp Neurol 486:267-280.

Wiesner S, Legate KR, Fassler R (2005) Integrin-actin interactions. Cell Mol Life Sci 62:1081-1099.

Xiao P, Bahr BA, Staubli U, Vanderklish PW, Lynch G (1991) Evidence that matrix recognition contributes to stabilization but not induction of LTP. NeuroReport 2:461-464.

Yamada KM, Even-Ram S (2002) Integrin regulation of growth factor receptors. Nature Cell Biol 4:E75-E76.

Yuan XB, Jin M, Xu X, Song YQ, Wu CP, Poo MM, Duan S (2003) Signalling and crosstalk of Rho GTPases in mediating axon guidance. Nat Cell Biol 5:38-45.

Zhang H, Webb DJ, Asmussen H, Niu S, Horwitz AF (2005) A GIT1/PIX/ $\mathrm{Rac} / \mathrm{PAK}$ signaling module regulates spine morphogenesis and synapse formation through MLC. J Neurosci 25:3379-3388. 